\title{
Stimulus-response curves as descriptors of corticospinal tract function: A functional imaging guided transcranial magnetic stimulation study in normal subjects and patients with subcortical stroke
}

\section{Sasan Ghinani}

\author{
Faculty of Graduate Studies \\ School of Neurological Sciences \& Neurosurgery \\ McGill University \\ Montreal, Quebec, Canada
}

September 2009

A thesis submitted to McGill University in partial fulfillment of the requirements of the degree of

Master of Science

(C) 2009 Sasan Ghinani. All rights reserved. 


\section{PREFACE}

\section{Structure of Thesis}

This thesis is formatted into chapters. The first chapter includes a background to the subject of structure, function and morphology of the corticospinal tract as well as the method of transcranial magnetic stimulation. This chapter also delineates the rationale, objectives and hypothesis for the presented study. The second chapter describes the methods and procedures used for the collection and analysis of data pertaining to this study. The third chapter presents the results of this research. The fourth and final chapter includes a discussion, conclusion as well as future recommendations on the subject of dose-response curves as predictors of corticospinal tract integrity.

\section{Contribution of Authors}

This research was done under the supervision of Dr. Alexander Thiel, who contributed to the design of this study, as well as the interpretation of results and the revision of this thesis. I, Sasan Ghinani, was in charge of devising methods, administration of TMS, data analysis and statistical analysis. Subject and patient recruitment, scheduling and behavioral tests were carried out by Sharon Shapiro, a research coordinator at the Jewish General Hospital, Montreal, Quebec, Canada. MRIdata used for this study were acquired at the Brain Imaging Centre of the Montreal Neurological Institute, Montreal Quebec Canada. 


\section{Acknowledgements}

I would like to acknowledge the Lady Davis Institute for Medical Research, the Jewish General Hospital and the graduate program of Neurological Sciences and Neurosurgery at McGill University. I would like to specifically thank the MRI lab technicians at the Montreal Neurological Institute and my Master's Advisory Committee: Dr. Hemant Paudel, Dr. Jeffry Minuk and Dr. Joyce Fung. I would especially like to acknowledge to work and support of the Neuroplasticity Lab at the JGH: Basia Radlinska, Michael Sidel, Sarah Grant, Caroline Paquette, Sharon Shapiro and our supervisor Dr. Alexander Thiel. This research was funded by CIHR. 


\begin{abstract}
Motor evoked potentials (MEP) elicited by transcranial magnetic stimulation (TMS) are used to asses corticospinal tract (CST) function in clinical practice. Advancements in technology have increased TMS precision yet clinical protocols do not reflect the gain in precision required for neuroscientific research. The aim of this study was to determine whether parameters extrapolated from MEP responses accurately reflect CST function. TMS was administered to healthy controls and acute subcortical stroke patients. A sigmoid-shaped dose-response curve was observed in control subjects and patients with lesions outside the CST. Relative amplitude of MEPs is the best descriptor of CST integrity. Absence of a sigmoid relationship indicates CST impairment.
\end{abstract}




\begin{abstract}
Abrégé
Les potentiels évoqués moteurs (PEM) élicités par la stimulation magnétique trancrânienne (SMT) sont utilisés en clinique pour évaluer la fonction du faisceau corticospinal (FCS). Les avancements technologiques ont amélioré la précision de la SMT. Toutefois, les méthodologies cliniques ne reflète pas la précision requise pour la recherche neuroscientifique. Le but de cette étude était d'analyser les caractéristiques des courbes de doses-effets des PEM pour déterminer s'ils reflètent la fonction du FCS. La SMT a été appliquée chez des sujets sains ainsi que chez des patients ayant subi un accident vasculaire cérébral (AVC). Une relation sigmoïde est présente dans les courbes de doses-effets chez les sujets sains et chez les patients ne présentant pas de lésions directement sur le FCS. De plus, l'amplitude absolue n'est pas un bon descripteur de l'excitabilité cortical à cause de la grande variabilité interindividuelle. L'absence d'une relation sigmoïde indique une déficience grave de la FCS.
\end{abstract}




\section{TABLE OF CONTENT}

PREFACE

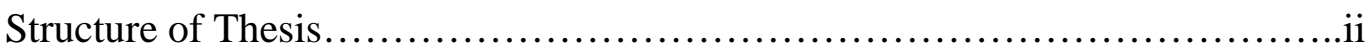

Contribution of Authors..........................................................ii

Acknowledgements ........................................................

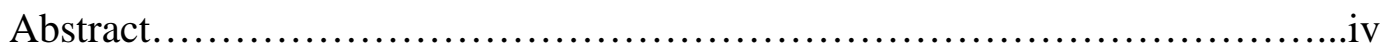

Abrégé.........................................................................

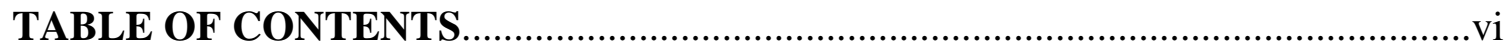

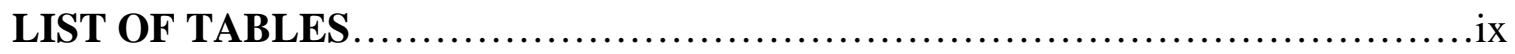

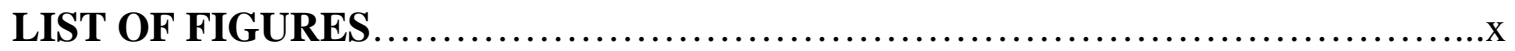

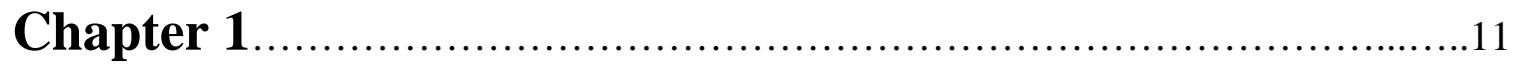

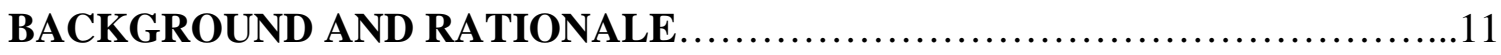

1.1 The Pyramidal Tract.................................................... 12

1.2 Motor Evoked Potential................................................13

1.3 Transcranial Magnetic Stimulation....................................... 14

1.4 Motor Threshold...................................................... 15

1.5 TMS in Clinical Neuroscience.........................................16

1.6 Limitations of Previous TMS Studies.....................................18

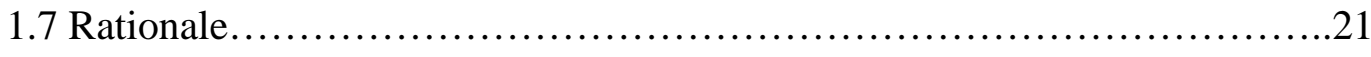

1.7.1 Research Questions.........................................22

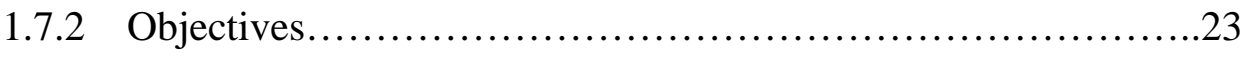

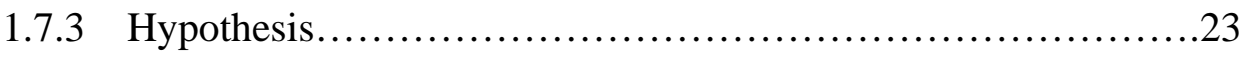


Chapter 2 .

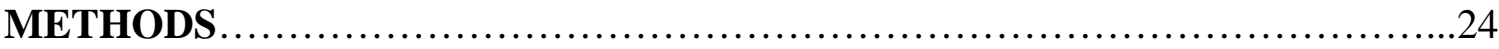

2.1 Subject.......................................................25

2.1.1 Inclusion Criteria..........................................25

2.1.2 Exclusion Criteria.........................................25

2.1.3 Control Subjects.......................................26

2.2 Recruitment.....................................................26

2.3 Procedures.......................................................... 26

2.3.1 Patient Procedures........................................26

2.3.2 Control Subject Procedures...................................27

2.4 Behavioral Testing..............................................28

2.4.1 Rivermead Motor Assessment...............................28

2.4.2 Nine-Hole Peg Test.......................................28

2.5 Magnetic Resonance Imaging.......................................29

2.6 Neuronavigation................................................ 30

2.7 Transcranial Magnetic Stimulation...................................31

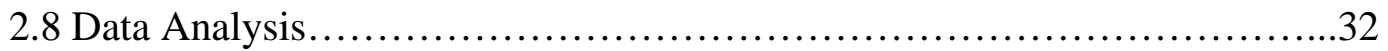

2.8.1 MRI Analysis........................................... 32

2.8.2 MEP Analysis........................................... 33

2.9 Statistical Analysis.............................................. 34

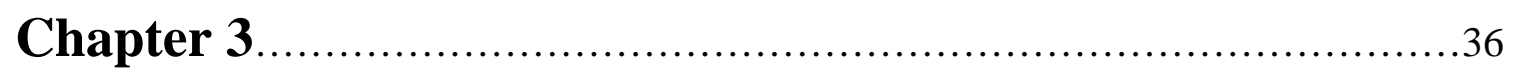

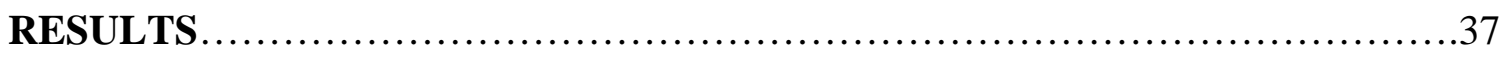

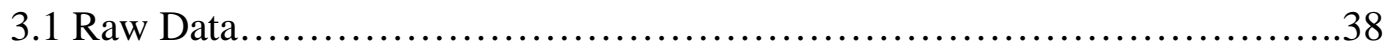


3.2 Amplitude............................................................ 41

3.2.1 Amplitude Plots.............................................41

3.2.2 Statistical Analysis of Amplitude...................................44

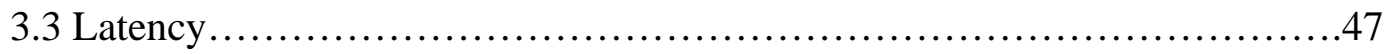

3.3.1 Latency Plots.............................................. 47

3.3.2 Statistical Analysis of Latency...................................51

3.4 Motor Threshold..................................................... 52

3.5 Pooled Data Analysis..................................................56

3.6 Relation of MEP Parameters to Motor Function..........................58

3.7 Influence of Stimulus Order on MEP Dose-Response Curve Parameters......59



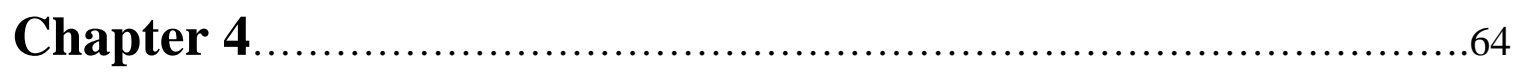

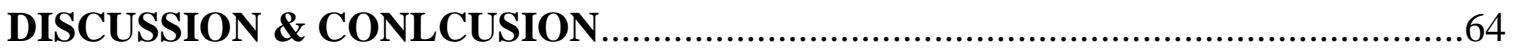

4.1 Amplitude...........................................................65

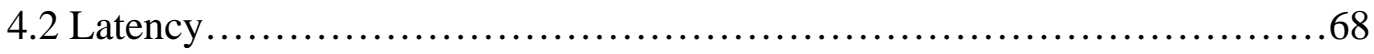

4.3 Methods of Determining Motor Threshold..................................69

4.4 Effect of Stimulation Order...........................................69

4.5 Hypothesis Tested................................................. 70

4.6 Future Applications................................................... 71

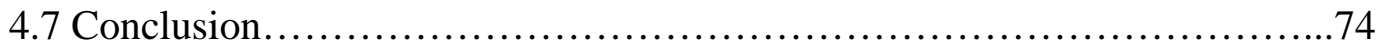

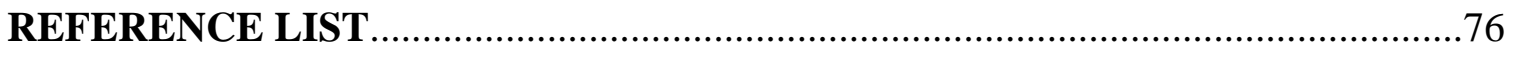




\section{LIST OF TABLES}

Table 1: Raw Data Collected from Control Subjects...............................39

Table 2: Raw Data Collected from Non-CST Affected Stroke Patients..................40

Table 3: Raw Data Collected from CST Affected Stroke Patients.....................40

Table 4: Prevalence of Sigmoid Fit in CST Affected Stroke Patients..................44

Table 5: Summary of behavioral motor tests.................................58 


\section{LIST OF FIGURES}

Figure 1: Equation of sigmoid function................................... 34

Figure 2: TMS results displaying sigmoid relationship of dose-response curve..........38

Figure 3: Amplitude of MEPs in control group..............................42

Figure 4: Amplitude of MEPs in Non-CST group.............................43

Figure 5: Amplitude of MEPs in CST group................................43

Figure 6: Difference in amplitude. Non-Affected - Affected ....................45

Figure 7: Pair-wise comparison of amplitudes...............................46

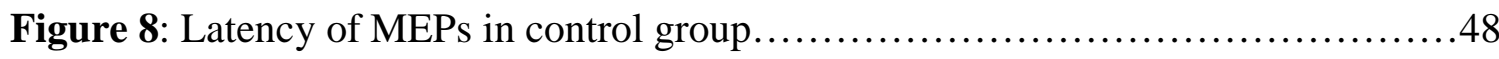

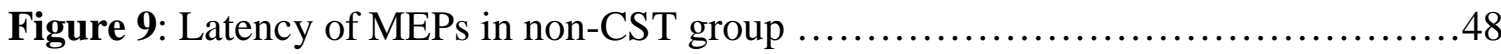

Figure 10: Latency of MEPs in CST group..................................49

Figure 11: Average latencies of Control Group..............................49

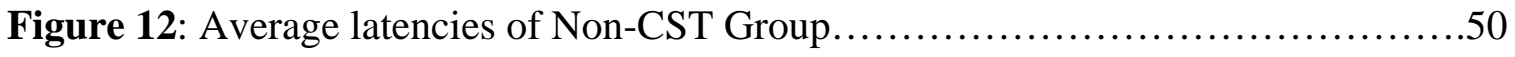

Figure 13: Average latencies of CST Group..................................51

Figure 14: Difference in Latency. Non-Affected - Affected .....................52

Figure 15: Difference in Resting Motor Threshold. Non-Affected - Affected..........53

Figure 16: Difference in Extrapolated Motor Threshold. Non-Affected - Affected.....55

Figure 17: Relationship between Resting Motor Threshold and the Extrapolated Motor

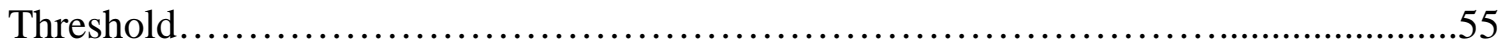

Figure 18: Pooled Non-CST and Control group compared to CST group..............56

Figure 19: Amplitude, EMT and latency compared in regular vs inverse protocol.......60

Figure 20: Recommendations for the use of TMS dose response curves..............72 
Chapter 1

\section{BACKGROUND AND RATIONALE}




\subsection{The Pyramidal Tract}

The pyramidal tract is the major pathway that controls voluntary movement (Netter et al., 2002). The tract consists of axons that originate from the pyramidal cells of the primary motor cortex (M1), and descend to peripheral motor neurons in the spinal cord. These axons descend from M1, through the white matter of the hemisphere, then pass through the posterior limb of the internal capsule and continue through the midbrain, the pons and through the medullary pyramids. At the level of the medulla, $90 \%$ of the axons cross to form the lateral corticospinal tract (CST), while 10\% remain ipsilateral and form the corticonuclear tract (also known as the corticobulbar tract) (Greenstein \& Greenstein, 2000). Fibers of the corticonuclear tract are mainly involved with the control of facial and oral muscles (Greenstein \& Greenstein, 2000), whereas fibers of the CST will carry information from the brain to the spinal cord and form synapses with the motorneurons of peripheral skeletal muscles (Hendelman, 2006; Netter et al., 2000; Greenstein \& Greenstein, 2000). Fibers of the CST are mainly involved the fine movement control (Hendelman, 2006). Lesion studies in rats showed that damage to the CST caused persistent deficits in fine control and skilled forelimb movement (Kanagal \& Muir, 2007; Anderson, Gunawan \& Steward, 2005).

In humans, advancements in neuroimaging techniques allow us to view the structural integrity of the CST. With diffusion tensor imaging (DTI), fiber tracking using seed regions permits the mapping of individual fiber bundle projections. This modality yields morphological information but provides no information about the functional integrity of individual tracts (Zhang et al., 2008).

Measuring motor evoked potentials and conduction velocity gives insight into the functional integrity of fiber tracts. These parameters are used as predictors of motor 
recovery for patients who have suffered CST damage (Brouwer \& Schryburt-Brown, 2006).

\subsection{Motor Evoked Potential (MEP)}

The functional integrity of the pyramidal tract is assessed by stimulating the motor cortex and measuring a response in the muscle that is controlled by the stimulated motor neurons. This response is recorded with electromyography (EMG) and is called a motor evoked potential (MEP) (Pascual-Leone et al., 2002). The amplitude and latency of MEPs are used clinically to assess CST function (Muellbacher et al., 2000; Brouwer \& Schryburt-Brown, 2006). MEP amplitude, measured in microvolts $(\mu \mathrm{V})$ or millivolts $(\mathrm{mV})$, correlates with the number of motor units recruited in a target muscle. Therefore, it is an indirect measure of the number of fibers that are stimulated (Muellbacher et al., 2000). The latency of MEPs, measured in milliseconds (ms), is the time needed for the stimulus to travel from M1 to the target muscle. Measures of conduction velocity can be derived from the latency of MEPs (Pascual-Leone et al., 2002; Peterson, Pyndt \& Nielsen, 2003).

The amplitude and latency of MEPs are affected by a number of variables ranging from inter-individual differences, drugs, fatigue and pre-stimulation muscle contraction, to motoneuron diseases, neurodegenerative disorders, CST lesions and acute trauma (Pascual-Leone et al., 2002).

MEPs characteristics permit the quantification of CST impairment in several neurological diseases and differentiation of central from peripheral motor neuron damage (Pascual-Leone et al., 2002). Furthermore, MEPs are used to monitor the progression of 
disease and functional recovery from a lesion during rehabilitation as well as to predict disease outcomes (Pascual-Leone et al., 2002).

\subsection{Transcranial Magnetic Stimulation}

Transcranial magnetic stimulation (TMS) is a non-invasive method to stimulate the cerebral cortex through the skull with an electromagnetic coil. It is a safe and practical way to elicit MEPs in a target muscle (Pascual-Leone et al., 2002).

TMS is performed using a hand-held coil that is energized by a large capacitor. When rapidly changing current flows through the coil, a magnetic field is induced orthogonally to the plane of the coil (Bueche, 1988). Since this flux exists only when currents in the coil are changing, TMS pulses are very brief (Cowey, 2005). This rapidly changing field induces an electric current in the brain (Jahanshahi \& Rothwell, 2000).

The magnetic field passes unimpeded through the scalp and the skull, providing a non-invasive method of stimulating the brain (Jahanshahi \& Rothwell, 2000). The magnetic field is however diminished over distance and as a result, only the superficial layers of the cortex can be stimulated. Depending on the make of the coil, TMS can stimulate approximately $2 \mathrm{~cm}$ into the brain (Cowey, 2005). This short-lasting, superficial

stimulation is enough to depolarize neurons and cause a chain of electrical signaling events within cells and chemical signaling between adjacent cells to create an action potential in the target muscle (Cowey, 2005). The motor cortex, in particular the arm and hand regions are a prime target area for TMS research due to its accessibility and the relative ease of recording results.

The specificity of the stimulation depends on the type of coil being used. In a circular coil, the magnetic flux produced by the alternating current through the loop will 
induce a circular magnetic field parallel to the coil. A circular coil will thus have a large target area that covers large portions of the cortex at a time due to the broad circular electric field maximum obtained (Cacioppo, Tassinary \& Berntson, 2007). In a figure of 8 coil, two circular coils are positioned in a way where their magnetic fields are constructively combined at the point where the two coils meet (Cacioppo, Tassinary \& Berntson, 2007), generating a cone-shaped field. Magnetic field strength is strongest at the centre of the cone. This coil arrangement allows relatively selective stimulation of a target area of approximately $1 \mathrm{~cm}$ in diameter. For all experiments in this study, a focal figure of 8 TMS coil was used.

Recent advancements in technology have increased the precision of coil positioning, and recording systems. Images obtained through functional magnetic resonance imaging (fMRI) are used in stereotaxic neuronavigation systems to guide TMS coils for precise stimulation (Herwig et al., 2001; Schonfeldt-Lecuona et al., 2005; Sparing et al., 2008). In addition to clinical uses, TMS is now used as a tool for scientific research allowing precise mapping of the cortical representations of muscles and changes in their spatial extent following cortical lesions (Pascual-Leone et al., 2002). However, parameters used in clinical electrophysiological protocols to quantify single MEPs do not always reflect this additional gain in precision which is of interest in neuroscientific research (Tranulis et al., 2006) These technological innovations necessitate innovations in data analysis methodology (Tranulis et al., 2006).

\subsection{Motor Threshold (MT)}


In order to evoke a motor potential, a certain level of stimulus intensity is required to depolarize motor neurons. This level of stimulus intensity is referred to as the motor threshold (MT) and can be measured at a subject's resting state (RMT) or during muscle contraction (Pascual-Leone et al., 2002; Di Lazzaro et al., 2008). MT is defined as the minimum stimulus intensity of TMS at which a MEP of defined size can be recorded (Duque et al., 2005; Di Lazzaro et al., 2008; Malcolm et al., 2006; Pascual-Leone et al., 2002). It is used as an indirect measure of cortical excitability (Muellbacher et al., 2000). MT varies greatly from one individual to the next and can be altered by the same factors that affect MEP amplitude. Nevertheless, MT is reasonably consistent in healthy subjects over time and offers an adequate measure of one's receptiveness to TMS (Pascual-Leone et al., 2002).

In clinical and research settings, dosage of TMS intensities are typically set as a percentage of MT (Muellbacher et al., 2000; Liepert et al., 2000; Woldag et al., 2004). Although it holds an important roll in clinical and neuroscientific protocols, there are only operationally defined procedures for establishing MT (Pascual-Leone et al., 2002; Tranulis et al., 2006). This constitutes a critical setback for clinical and research applications of TMS and will be discussed in greater detail in section 1.6 (Limitations of Previous TMS Studies).

\subsection{TMS in Clinical Neuroscience}

Focal stimulation of the motor cortex reveals information about cortical excitability, CST function and plastic changes that occur following disease or injury (Pascual-Leone et al., 2002). 
Brower \& Schryburt-Brown (2006) used TMS to measure cortical excitability and related these measurements to hand function in chronic stroke patients. Cortical excitability was assessed by absolute MEP amplitude and hand function was measured by finger tapping frequency, peg placing time and grip strength. They concluded that abnormalities in cortical excitability post stroke are positively correlated with a decrease in hand function. This is an example of the types of results that have come from a standard set up wherein TMS is administered to sub-acute and chronic stroke patients using a figure of 8 coil. MEPs were recorded from the first dorsal interosseous (FDI) muscle and stimulus intensity was set at $20 \%$ above MT. For this experiment, MT was defined as the lowest intensity required to elicit 5 MEPs with an amplitude of $50 \mu \mathrm{V}$ or higher out of 10 consecutive stimuli (Brouwer \& Schryburt-Brown, 2006).

TMS has also been used as a means to examine differences in bilateral connections and the phenomena of inhibition and compensation between hemispheres following injury. Muellbacher et al. (2000) conducted an experiment to test whether unilateral hand muscle activation had an effect on the excitability of the ipsilateral cortex of healthy subjects. Results indicated that there is an effect on the excitability of the ipsilateral M1 due to voluntary unilateral hand muscle activation (Muellbacher et al., 2000). Caramia et al. (2000) found that ipsilateral MEPs can be elicited in patients and in some healthy controls in an area $3 \mathrm{~cm}$ lateral and $3 \mathrm{~cm}$ anterior to the optimal site for eliciting contralateral MEPs. It was suggested that these ipsilateral corticospinal tracts become more active following cerebral damage (Caramia et al., 2000).

Foltys et al. (2003), aimed to uncover the mechanisms leading to rapid and firstrate recovery of motor function after stroke. Results of fMRI showed bilateral activation of patients with rapid and nearly complete recovery of motor function. However, TMS 
data failed to show ipsilateral motor pathways; therefore recovery was thought to be based on existing bilateral motor pathways spared by injury (Foltys et al., 2003). This study supports the hypothesis of pre-existing ipsilateral connections however cannot offer an explanation for the mechanisms of activation of these pre-existing pathways.

In light of the findings described in previous TMS studies, there are a few points to take into account when preparing a TMS protocol. For one, considering the evidence of inhibition and compensation between hemispheres after injury, it is important to perform tests on patients in the acute stage. Much research is devoted to the mechanisms of stroke recovery in chronic stroke patients, however investigating the mechanisms occurring in the acute stage post-stroke is less common (Brouwer \& Schryburt-Brown, 2006). Secondly, due to evidence of bilateral connections, it is important to insure that both hands are at a state of rest when performing TMS on one side or the other (Foltys et al., 2003; Muellbacher et al., 2000; Caramia et al. 2000).

\subsection{Limitations of Previous TMS Studies}

One of the most variable aspects of conventional focal TMS research is the way in which the examiners locate the area of maximal MEP response (hotspot) (Sparing et al., 2008). Most often, examiners will search for this area by combing over the scalp of the subject with the coil while sending pulses and monitoring the reaction (Mullbacher et al., 2000; Johansen-Berg et al., 2002; Woldag et al., 2004; Renner et al., 2005). The precision of this method is completely dependent on the experience of the examiner. In many studies, the hotspot is marked with a felt-tipped pen directly on the scalp of the subject, where the highest MEP amplitude was recorded (Muellbacher et al., 2000; Liepert et al., 2000). 
Other studies use a bathing cap placed over the head of the subject. The cap normally has $1 \mathrm{~cm}^{2}$ grids, which offers the possibility of marking hotspots and quantifying their location using coordinates (Traversa et al., 1999; Renner et al., 2005; Stinear et al., 2007). The caps can also be kept and offer some reproducibility for follow up sessions, assuming it is placed in the exact same position on the head. Although this method is more reliable than a pen mark on the scalp, both methods have limitations that can be surpassed using a stereotaxic neuronavigation system.

A focal coil stimulates a cortical area of approximately $1 \mathrm{~cm}$ in diameter. Therefore, any subtle movement of the coil can cause a change in induced current density and direction owing to the variation in shape of the cortical gyri (Sparing et al., 2008). Once the location of the hotspot is determined, it is important to be able to detect whether, through the course of the TMS session, the coil is still positioned at the exact same point. The duration of TMS sessions can last over an hour depending on the research protocol. Within this time it is natural that a subject may sway or move enough to hinder the precision of the coil placement (Tranulis et al., 2006; Sparing et al., 2008). Furthermore, an examiner or a mechanical arm which holds the coil may not be able to stay completely still for the duration of the session. A controlled parameter must be introduced to correct for the possible movements of the patient, the coil or both, to ensure the coil remains over the area of maximum activation for the duration of the session.

With the conventional methods of TMS, one can only speculate as to the anatomical features of the cortex beneath the hotspot. It would be advantageous to know exactly what area of the brain is being stimulated by the coil, to see if that area changes over time and for increased precision of coil placement (Sparing et al., 2008). 
Another area of discrepancy within TMS studies is the way in which motor threshold (MT) is obtained. As mentioned, dosage of TMS intensities are typically set as a percentage of MT (Muellbacher et al., 2000; Liepert et al., 2000; Woldag et al., 2004), therefore it is a crucial parameter for TMS studies. Motor threshold obtained by TMS is an objective measure of cortical excitability.

Tranulis et al. (2006) offered a comparative analysis of the three most commonly used methods of determining MT. These include the Rossini-Rothwell method, which consists of lowering the stimulus intensity until only 5 responses out of 10 consecutive trials are recorded. A valid response is one that has an amplitude of at least $50 \mu \mathrm{V}$. The stimulus intensity at which this is reached is considered to be the motor threshold. The Mills-Nithi technique calculates the MT as the mean of an upper threshold (10 responses out of 10 consecutive trials) and the lower threshold ( 0 responses out of 10 consecutive trials). Once again, a valid response is one in which the amplitude of the action potential is at least $50 \mu \mathrm{V}$. The third proposed method of determining MT is a mathematically derived algorithm based on a sigmoid function fitted to the dose-response curve of the MEPs. This method suggests that the intensity at the center of the sigmoid function $\left(\mathrm{I}^{1 / 2}\right)$ is the MT (Tranulis et al., 2006).

The first two methods do not use all the available data in calculating the MT. They are based on a stochastic definition and an arbitrary criterion of amplitude size (Tranulis et al., 2006). The analytical approach uses all available experimental data to estimate MT via curve-fitting and does not make an assumption of minimum MEP size. Thus theoretically it should be the most valuable means in predicting MT (Tranulis et al., 2006). In their comparative study however, Tranulis et al. (2006) found that there were no significant differences in the validity of MT between the three methods in normal 
subjects. Nonetheless, the Rossini-Rothwell method was placed above the other two in clinical practice on the account that it uses the least trials and hence takes less time to calculate. They also propose that the mean variability of all three methods is acceptable for clinical purposes, but may cause discrepancies in neuroscientific studies of cortical excitability. Recommendations were made for ulterior techniques for determining MT to be evaluated. Novel techniques should take into account "within-session factors" such as hotspot determination, movement of the subjects and the coil and control of muscle relaxation. The use of a stereotaxic neuronavigation system was offered as a solution to the aforementioned problems (Herwig et al., 2001; Tranulis el al., 2006; Sparing et al., 2008).

\subsection{Rationale}

Traditional parameters for analyzing MEP characteristics no longer reflect the precision obtained by neuronavigated TMS (Herwig et al., 2001; Tranulis el al., 2006; Sparing et al., 2008). New technologies offer alternative solutions to the problems of hotspot location, subject-coil movements, identification and quantification of coil positioning, MT determination, and the possibility of reliably eliciting MEPs with very low amplitudes (<50 $\mu \mathrm{v}$ ) (Herwig et al., 2001; Tranulis el al., 2006; Sparing et al., 2008). In light of these new progressions, recent studies have looked at MEP dose-response curves in normal controls with the goal of extracting novel parameters for the measurement of cortical excitability and CST function (Luft et al., 2001; Tranulis et al., 2006; Malcolm et al. 2006; Van Kuijk et al., 2008) Results show that a sigmoid function can reliably be reproduced in dose-response curves of healthy control subjects (Luft et al., 2001; Tranulis et al., 2006; Malcolm et al. 2006; Van Kuijk et al., 2008). It remains 
unclear if parameters from these dose response curves can offer new information with regards to cortical excitability and fiber tract integrity. Moreover, it has yet to be determined if this relationship is altered in patients with damage to the CST. The present study recorded dose-response curves in patients with subcortical stroke as a model for CST impairment.

A specific aim of this study was to apply previously described methods of dose response function to normal controls of varying ages in order to determine if new parameters can reliably be derived and to determine absolute and relative (left-right differences) values. In addition, this study aimed to apply these methods to patients with subcortical strokes and different extent of CST damage in order to determine: 1) if this relationship still holds for lesioned CST and 2) if the parameters are associated with behavioral measures of CST function.

\subsubsection{Research Questions}

(1) Are there other electrophysiological parameters of CST function other than amplitude, latency and RMT?

(2) Can dose-response curves be recorded in subjects with damage to pyramidal tract?

(3) If yes, what do parameters derived from these curves tell us about pyramidal tract function?

(4) Do these parameters reflect altered CST function in patients with subcortical stroke? 


\subsubsection{Objectives}

The objectives of this study was to assess whether parameters extrapolated from MEP dose-response curves could be used as a measure of cortical excitability in normal subjects of varying ages. Furthermore, we aimed to elucidate whether these parameters reveal information that were different from established measures such as resting motor threshold and MEP-amplitude.

\subsubsection{Hypothesis}

It was hypothesized that:

(1) A sigmoid function could reliably be fitted to dose-response curves of normal subjects and subcortical stroke patients.

(2) Parameters derived from these dose-response curves could be used as markers for CST function and cortical excitability in normal subjects as well as subcortical stroke patients. 
Chapter 2

\section{METHODS}




\subsection{Subjects}

The patient group was recruited from the acute stroke unit of the Jewish General Hospital (JGH) in Montreal, Quebec. All subjects gave a written informed consent to participate in this study, approved by the ethics committees of McGill University Faculty of Medicine, the JGH and the Montreal Neurological Institute (MNI).

\subsubsection{Inclusion Criteria}

Patients were entered into the study between 5 and 14 days after a first ever subcortical ischemic stroke affecting upper extremity motor function. Competency judged by the treating physician was a prerequisite for inclusion. Subcortical stroke patients included all those with infarcts in the white matter, the basal ganglia or the internal capsule. All patients received a diagnostic CT or MRI scan to assess infarct location. Based on these scans, patients were sub-divided into two groups: CST and NonCST groups. Patients in the CST group had a lesion that is directly affecting the CST. Patients in the Non-CST group had a subcortical lesion that is not directly in contact with the CST.

\subsubsection{Exclusion Criteria}

All patients with evidence of alcoholism, other drug abuse or taking psychoactive medications were excluded from the study. Participants with cardiac pacemakers or any other implanted ferromagnetic materials were also excluded from the study. Further exclusion criteria include claustrophobia, a history of epilepsy, neurodegenerative and psychiatric diseases (such as major depression, schizophrenia or bipolar disorder) and patients with grave upper extremity motor impairment. Impairment was assessed by a 
registered nurse using the Rivermead Motor assessment and the Nine Hole Peg Test. (Behavioral tests are delineated in section 2.4).

\subsubsection{Control Subjects}

As a control group, subjects with similar age and cardiovascular risk profile were investigated. These participants must not have suffered a stroke or display signs of subcortical lesions. Controls may or may not have had other health factors such as diabetes, hypertension or transient ischemic attack. Young healthy controls were recruited for the establishment of MRI and TMS protocols.

\subsection{Recruitment}

During the 19 months of recruitment, approximately 150 stroke patients were screened for eligibility and 63 met the inclusion criteria and were invited to participate. Of these 63 patients, informed consent was obtained from 15 subjects and of these, 13 subjects completed the study. One patient dropped out of the study due to claustrophobia, and one due to loss of interest.

Five age and risk matched controls were recruited from the stroke prevention program at the JGH and six young healthy control subjects were used to establish the methods and protocols.

\subsection{Procedures}

\subsubsection{Patient Procedures}

Patients admitted to the stroke unit at the JGH were screened by a neurologist for eligibility. All eligible patients were explained the study and consent form by research 
coordinator or the principal investigator. Once informed consent was obtained, the research coordinator fixed an appointment for the behavioral testing which took place at the JGH. Once the behavioral tests were complete, a date was scheduled for the MRI. The MRI took place at the McConnell Bain Imaging Centre of the MNI, Montreal, Canada. Transportation to and from the MNI was provided and patients were accompanied by a nurse during the transportation. Once the MRI was completed, an appointment was fixed for the TMS session. TMS was administered at the JGH. The time between the recruitment of the patient, behavioral tests, the MRI and TMS was dependant on whether the patient had returned home or was still an in-patient at the JGH. However the process from recruitment to TMS was always within 14 days post stroke. If the patient had already returned home from the hospital before the scheduled date for the behavioral tests, their transportation to and from the hospital was paid for by the funds allocated for this study.

\subsubsection{Control Subject Procedures}

Participants of the stroke prevention program at the JGH or spouses of the patients as well as any interested individuals who fit our inclusion criteria were explained the study and the consent form by a medical doctor. Once informed consent was acquired, the subjects were scheduled for the MRI at the McConnell Bain Imaging Centre of the MNI, Montreal, Canada. After the MRI was completed, another date was scheduled for the TMS session at the JGH. Subjects were reimbursed for the cost of their transportation to and from the MNI and the JGH. The time between the MRI and the TMS for control subjects varied between 1 and 7 days. No behavioral tests were performed by the control subjects. 


\subsection{Behavioral Testing}

\subsubsection{Rivermead-Motor Assessment}

The Rivermead-Motor Assessment is a standardized test used to measure motor dysfunction (Sommerfeld et al., 2004). In this test, a maximum score of 15 indicates no motor impairment and a score of 0 indicates severe motor impairment (Adams et al., 1997). All patients were assessed by a research coordinator prior to their fMRI. Patients with less than 6 points in the arm section of this assessment were excluded from the study because performance of the fMRI motor-task was not granted. (Please refer to section 2.5 in the Methods chapter for details about the fMRI motor task). All patients in our study were able to attain the minimum score and therefore none had to be excluded for this reason. Results of the Rivermead Motor Assessment were correlated with parameters of TMS dose-response curves.

\subsubsection{Nine-Hole Peg Test (NHPT)}

To further differentiate precision of motor performance, patients performed the NHPT under the supervision of trained research staff. The NHPT is commonly used as a measure of finger dexterity for stroke patients (Sommerfeld et al. 2004). It consists of placing nine pegs in a peg board using one hand in the least amount of time. Results are measure by the amount of time it takes to place all 9 pegs or by the number of pegs placed after 60 seconds (Chen et al., 2009). Results of this test were correlated with parameters of TMS dose-response curves. 


\subsection{Magnetic Resonance Imaging}

Magnetic resonance imaging was performed on a 3 Tesla Siemens Trio scanner at the McConnell Bain Imaging Centre of the MNI, Montreal, Canada. The MRI sequence was comprised of five parts: a 12 minute high resolution $\mathrm{T} 1$ weighted anatomical scan $(1 \times 1 \times 1 \mathrm{~mm}$ isotropic, interleaved slices, $\mathrm{TR}=23 \mathrm{~ms}, \mathrm{TE}=7.4 \mathrm{~ms})$, two 7 minute functional MRI protocols $(4 \times 4 \times 4 \mathrm{~mm}$ isotropic, descending slices, $\mathrm{TR}=1500 \mathrm{~ms}, \mathrm{TE}=$ 30ms) and two 12 minute diffusion weighted acquisitions. The DTI sequences were acquired as part of an ongoing parallel study and thus are relevant only to the extent that their acquisition added to the total time the subjects spent in the scanner. Total scanning time was one hour.

The f-MRI protocol comprised a cued active finger tapping task and a resting control state. For the duration of the fMRI acquisition, subjects heard a regular computer generated tone synched to the MRI produced by Presentation ${ }^{\circledR}$ software from Neurobehavioural Systems $\odot$. The duration of each tone was $10 \mathrm{~ms}$ and the interval of time between the beginning of one tone and the next was $1.5 \mathrm{~s}$. A finger-tapping apparatus constructed entirely of MR compatible material was used for the task. The apparatus consisted of a piston driven by pneumatic pressure to produce a constant resistance for the finger $(275 \mathrm{KPa})$. Thus, the FDI muscle was required to exert a defined force on the piston. The pneumatic pressure came from a non-breathable, medical air tank provided by MEGS Specialty Gases Inc.

The tone was relayed to subjects through headphones while they were in the scanner. Subjects were asked to tap their index finger in time with the tone during the active phases, signaled by the word "Start". Subjects were asked to remain still without tapping their finger during the resting phases, signaled by the word "Stop". Each phase 
was made up of 10 tones, lasted 15 seconds and was repeated 14 times. Subjects practiced before they entered the MRI to ensure they understood the task.

\subsection{Neuronavigation}

The Brainsight ${ }^{\mathrm{TM}}$ frameless stereotaxy system (Rogue Research) at the JGH enabled us to visualize the part of the cortex the coil is placed over (Sparing et al., 2008). It allowed accurate coil placement and the ability to save the coordinates of the stimulation site (Hughes \& John, 2003). Functional MR-images were superimposed and co-registered with the anatomical images to match the dimensions of one image to the other. The images were then loaded onto Brainsight ${ }^{\mathrm{TM}}$ Frameless software (version 1.5, build F2.3). A subject tracker which consisted of three retro-reflective markers on a headband was placed around the subject's head. Anatomical landmarks (the tip of the nose, the bridge of the nose, the left and right tragus and the vertex) were defined and registered with the MR-images of the subject on the screen. A Polaris camera system from Northern Digital Inc. was used to capture the position of the trackers. This optical tracking system measured the 3D positions of reflective markers affixed to the subject's head and the TMS coil. Registering the anatomical landmarks on the subject's head determined the dimensions of the head and position of the landmarks with regards to the subject tracker. With this, the system computed the position of the subject's head is in space in real time and related this information to the MR-images on the monitor (Sparing et al., 2008).

The tracker on the coil allowed the system to compute the coil position with regards to the subject's head. Hence, when the coil was placed over the scalp of the subject, the computer monitor showed the position of the coil and the underlying area of 
cortex. The coordinates of the coil trajectories were saved and used as a guidance cue to keep the coil in the correct position or to reposition the coil in case the subject or examiner moved.

\subsection{Transcranial Magnetic Stimulation}

TMS was performed using a Magstim Rapid stimulator (MAGSTIM Ltd) with a $7 \mathrm{~cm}$ figure of 8 , hand held coil. Subjects were seated on a chair and asked to remain still in a comfortable position while keeping their hands relaxed and placed on their laps. No tasks had to be carried out by the subjects during TMS.

Surface EMG-electrodes were placed over the first dorsal interosseous (FDI) muscle of both hands with the different electrode over the muscle belly and the indifferent electrode over the tendon at the first metacarpo-phalangeal joint. The location of maximal stimulation, referred to as the "hotspot" was determined by placing the coil of the magnetic stimulator over the area of the motor cortex, representing hand movement as defined by the f-MR-image using the frameless stereotaxy system. This area was verified by manual systematic assessment over the scalp. TMS pulses were given over that area at $80 \%$ of maximum stimulator output and MEPs were recorded. The 3D spatial coordinates

of the coil over the hotspot were saved for each patient over both motor cortices. The coil was locked into place with a mechanical arm to minimize the chance of it moving during the TMS session.

Single magnetic pulses were delivered over the hotspot and MEPs were recorded from the FDI-muscle. Five pulses were delivered at each level of intensity starting at $100 \%$ maximum stimulator output and decreased in increments of 5\% until there were no evoked responses for five consecutive stimulations. Pulses were separated by a minimum 
of 10 seconds to avoid summation and carry-over effect. The times between stimuli varied by 2-4 seconds to ensure subjects were not expecting stimulation at a given interval of time. This procedure was performed for both hemispheres.

\subsection{Data Analysis}

\subsubsection{MRI Analysis}

Signals in fMR-images are based on the Blood Oxygen Level Dependant, or BOLD-effect. This effect depends on the relationship between neural activity and cerebral blood flow. Activated neurons require a quick source of glucose and oxygen (McIntire et. al, 2003). Therefore, oxyginated blood is delivered to neurons that are firing at a greater rate than inactive neurons. This response is called the hemodynamic response. Since the magnetic resonance of blood is different depending on its level of oxygination, using the BOLD effect it is possible to differentiate between active and inactive areas of the brain (McIntire et. al, 2003)

Images obtained from the functional scan were processed using FSL software through the FEAT analysis (6mm FWHM Gaussian filter smoothing kernel). The FSL FEAT program enabled us to create a model of expected brain activity based on the experimental design of our fMRI motor task. FEAT uses a multiple regression algorithm for the model-based analysis of fMRI data (Smith et al., 2007). With this analysis we were able to see what parts of the brain fit the expected model of activation (Makni et al., 2008). Images obtained by FEAT showed the areas of the brain that were working during the fMRI active task.

Functional MR-images were superimposed and co-registered with the high resolution morphological scan using the Multi-Modality Matching (MMM) Co- 
registration tool available in the VINCI 2.55 software in order to match the dimensions of one image to the other (Cizek et al., 2004). We used these images to determine the anatomical location of the hand representation on the motor cortex in each subject for the purposes of neuronavigation.

\subsubsection{MEP Analysis}

The system used for MEP data acquisition and analysis came from AD Instruments. The PowerLab 8/30, model number ML870 is a data acquisition system that comprises EMG and is suitable for neuroscientific research (Chen et al., 2008). It was used in conjunction with Scope ${ }^{\mathrm{TM}}$ software for Windows ${ }^{\circledR}$. These systems allowed us to record and save MEP data so that it was accessible for offline analysis. Amplitude and latency of MEPs were collected by going through the tracing of each MEP elicited after every TMS pulse. Amplitude was defined as the highest point (the peak) of the MEP and latency was defined as the time interval between the stimulus and the rising phase of the MEP.

The EMG high pass filter was set at $0.3 \mathrm{~Hz}$ and low pass filter at $100 \mathrm{~Hz}$. EMGs were recorded with a speed of 100 samples per 5 milliseconds. EMG recording was done in a live-feedback setup in both hands simultaneously while TMS was administered to one hemisphere at a time. With this setup, the examiners as well as the subject were able to see if there was any level of activity in the muscles being recorded. This was to insure that the muscles in both hands were relaxed while performing TMS. MEPs measured from muscles that were not relaxed prior to stimulation were discarded during data analysis. 


\subsection{Statistical Analysis}

Statistical analysis was performed using SigmaPlot 11 and SigmaStat 3.5 software (Systat Inc.). The sigmoid relationship of the dose-response curves were fitted using a least square fit procedure. A three parameter sigmoid equation was used (Fig. 1) and the total number of fits used in the graphs was 200.

Figure 1: Equation of sigmoid function

$$
y=\frac{a}{1+e^{-\left(\frac{x-x_{0}}{b}\right)}}
$$

Figure 1 represents the three parameter equation of the sigmoid function fitted to TMS dose-response curves. The three parameters include: a) the amplitude, b) the slope and $\mathrm{x}_{0}$ ) the position of the curve on the $\mathrm{x}$ axis.

To control for interindividual variability, we compared a patient's affected hemisphere to their healthy hemisphere. One way analyses of variance (ANOVA) were performed to assess the mean values of the differences in MEP amplitude, latency and RMT between the CST group, the Non-CST group and the control group. Differences were calculated as the value from the non-affected hemisphere minus the value from the affected hemisphere. In control subjects, we arbitrarily assigned the left and right hemispheres as healthy and affected, respectively.

A T-test was performed when comparing the difference in EMT of the Non-CST group and the control group. Three paired t-tests were performed to compare the MEP amplitude of the affected hemisphere to the MEP amplitude of the non-affected hemisphere. Since this test was performed for each of the three groups, a Bonferroni 
adjustment was used to correct for multiple comparisons. A Fisher Exact test was performed for the analysis of a contingency table comparing the presence or absence of a sigmoid fit in data acquired from affected and non-affected hemispheres of the CST group. The Fisher Exact test was best suited for this analysis considering the small sample size (Mehta \& Patel, 1983). 


\section{Chapter 3}

\section{RESULTS}




\section{Results}

Amplitude and latency were measured from the MEPs. Amplitudes were plotted against the percentage of maximum stimulator power to give a dose-response curve. Dose-response curves followed a sigmoid relationship and parameters from these curves were computed by non-linear curve fitting (Figure 2). A straight line was fitted to those data points defining the linear part of the sigmoid function. The intersection of this line with the abscissa (X-intercept) represents a similar measure of cortical excitability as resting motor threshold (RMT). However this measure is not only dependent on the position of the curve along the abscissa, it also influenced by the slope of the linear part of the curve. This extrapolated motor threshold (EMT) was mathematically derived and is thus not based on stochastic measures. Other extrapolated data included the slopeparameter of the sigmoid function, the slope of the linear function, the stimulator intensity at the half maximum of the sigmoid function, and the maximum MEP. 
Figure 2: TMS results displaying sigmoid relationship of dose-response curve.

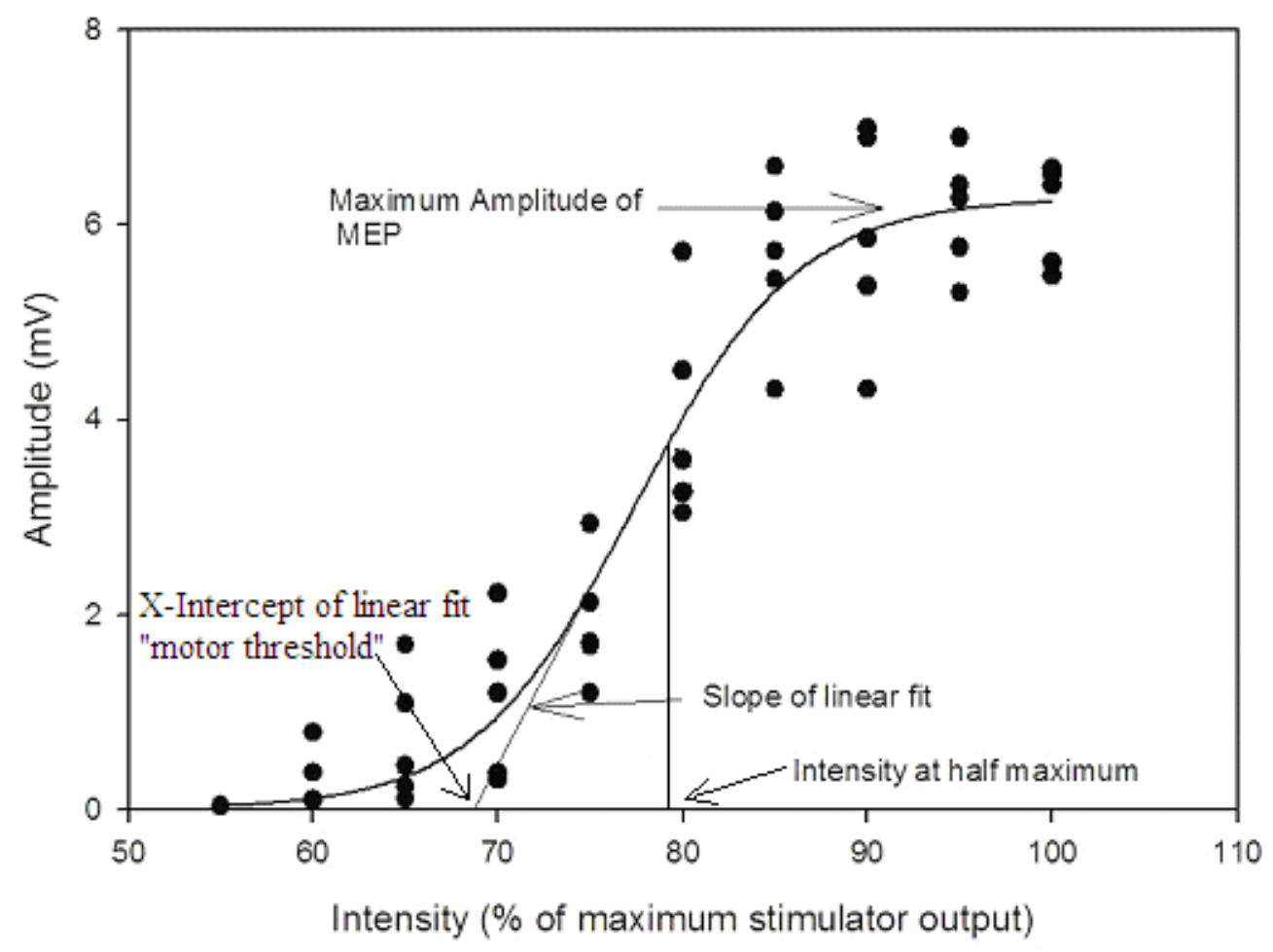

Figure 2 represents the sigmoid curve obtained from transcranial magnetic stimulation of the motor cortex corresponding to the FDI. The labels, Maximum Amplitude of MEP, Intensity at half maximum, Slope of linear fit, and X-intercept of linear fit or "extrapolated motor threshold" represent the determined parameters. The parameter referenced on the ordinate is Amplitude, in millivolts $(\mathrm{mV})$. Intensity, in percentage of maximum stimulator output, is referenced on the abscissa.

\subsection{Raw Data}

The following parameters were determined for each subject. (See tables 1, 2 and 3)

i. $A_{\max }$ (maximum MEP amplitude, height parameter a from sigmoid fit)

ii. $b_{\text {sig }}$ (slope parameter $b$ from sigmoid fit) 
iii. $\mathrm{I}_{1 / 2}$ (Intensity at half maximum, parameter $\mathrm{X}_{0}$ from sigmoid fit)

iv. $\mathrm{t}_{1 / 2}$ (average latency from MEPs within $\mathrm{I}_{1 / 2}+/-5 \%$ )

v. $t_{\max }$ (average latency from MEPs at the highest TMS intensity output)

vi. $b_{\text {lin }}$ (slope parameter $b$ from linear fit)

vii. $\mathrm{I}_{0}$ (Intensity at amplitude 0 extrapolated from linear fit: $\mathrm{I}_{0}=-\mathrm{a} / \mathrm{b}_{\text {lin }}$, with a being the intercept of the regression line referred to as EMT)

viii. $\mathrm{r}^{2}$ (Goodness of fit of the sigmoid curve)

Table 1: Raw Data from Control Subjects

\begin{tabular}{|l|c|c|c|c|c|c|c|c|c|c|c|}
\hline Subject & $\begin{array}{l}\text { Side } \\
\text { of } \\
\text { TMS }\end{array}$ & $\begin{array}{l}\text { Affected } \\
\text { Hem }\end{array}$ & Age & Amax & Bsig & I1/2 & T1/2 & Tmax & Blin & I0 & $\mathrm{r}^{2}$ \\
\hline A.T & right & none & 39 & 0.4101 & 3.229 & 92.762 & 24.37 & 24.44 & 0.0291 & 85.787 & 0.6394 \\
\hline M.S & right & none & 30 & 1.6425 & 4.529 & 87.535 & 24.07 & 23.925 & 0.0857 & 77.964 & 0.7862 \\
\hline S.G & right & none & 22 & 5.3618 & 5.8188 & 87.453 & 24.43 & 22.58 & 2.171 & 75.131 & 0.9127 \\
\hline C.S & right & none & 21 & $1.05 \mathrm{E}+08$ & 8.7185 & 263.71 & n/a & 22.49 & 0.0675 & 89.27 & 0.6728 \\
\hline L.K & right & none & 24 & 4.5221 & 14.208 & 99.203 & 22.68 & 22.68 & 0.0715 & 68.2 & 0.5214 \\
\hline B.B & right & none & 69 & 1.5742 & 11.123 & 99.632 & 25.03 & 25.03 & 0.0343 & 76.83 & 0.949 \\
\hline B.B & left & none & 69 & 4.3736 & 6.0295 & 83.199 & 25.05 & 24.81 & 0.1772 & 70.84 & 0.944 \\
\hline L.F & right & none & 57 & 2.0917 & 7.6248 & 89.508 & 26.71 & 25.1 & 0.0672 & 73.976 & 0.925 \\
\hline L.F & left & none & 57 & 254856 & 4.7403 & 157.2 & n/a & 25.79 & 0.2482 & 94.177 & 0.000 \\
\hline K.R & right & none & 59 & 3.696 & 3.054 & 88.752 & 30.21 & 28.64 & 0.2955 & 82.493 & 0.999 \\
\hline K.R & left & none & 59 & 4.3096 & 5.7492 & 80.566 & 29.65 & 28.54 & 0.1822 & 68.732 & 0.954 \\
\hline C.L & right & none & 75 & 3.0049 & 16.011 & 72.271 & 25.74 & 23.02 & 0.0453 & 39 & 0.955 \\
\hline C.L & left & none & 75 & 1.6605 & 4.9486 & 78.733 & 24.83 & 25.38 & 0.0824 & 68.66 & 0.844 \\
\hline P.D & right & none & 65 & 1.2886 & 7.6209 & 75.152 & 26.18 & 24.63 & 0.0412 & 59.5 & 0.877 \\
\hline P.D & left & none & 65 & 2.623 & 6.784 & 69.641 & 27.7 & 25.31 & 0.0961 & 55.99 & 0.770 \\
\hline
\end{tabular}


Table 2: Raw Data Stroke Patients where CST was Not Affected

\begin{tabular}{|l|c|c|c|c|c|c|c|c|l|l|l|}
\hline Subject & $\begin{array}{l}\text { Side } \\
\text { of } \\
\text { TMS }\end{array}$ & $\begin{array}{l}\text { Affected } \\
\text { Hem }\end{array}$ & Age & Amax & Bsig & I1/2 & T1/2 & Tmax & Blin & I0 & $r^{2}$ \\
\hline P.M & right & right & 75 & 1.2979 & 3.0104 & 76.185 & 26.62 & 24.9 & 0.1013 & 69.789 & 0.696 \\
\hline P.M & left & right & 75 & 2.833 & 3.3564 & 73.077 & 25.11 & 24.12 & 0.2034 & 66.102 & 0.939 \\
\hline R.W & right & right & 78 & $6.96 \mathrm{E}+07$ & 8.786 & 265.13 & n/a & 22.95 & 0.0633 & 88.89 & 0.963 \\
\hline S.D & right & left & 49 & $1.45 \mathrm{E}+08$ & 4.4021 & 181.93 & n/a & 25.34 & 0.1046 & 94.578 & 0.658 \\
\hline S.D & left & left & 49 & 10541217 & 5.1558 & 195.99 & n/a & 25.43 & 0.1719 & 93.491 & 1.00 \\
\hline D.H.A & right & right & 82 & $1 \mathrm{E}+07$ & 17.081 & 383.93 & n/a & 26.66 & 0.0349 & 81.74 & 0.698 \\
\hline D.H.A & Left & right & 82 & 1.431 & 0.2105 & 80.229 & 27.51 & 26.76 & 2.1946 & 79.91 & 0.924 \\
\hline J.B & Right & left & 64 & 3.4458 & 15.103 & 100.93 & 26.75 & 26.75 & 0.0566 & 70.5 & 0.886 \\
\hline J.B & Left & left & 64 & 6.7011 & 7.4747 & 95.473 & 28.21 & 27.59 & 0.2164 & 79.95 & 0.995 \\
\hline
\end{tabular}

Table 3: Raw Data Stroke Patients where CST was Affected

\begin{tabular}{|l|c|c|c|c|c|c|c|c|c|c|c|}
\hline Subject & $\begin{array}{l}\text { Side } \\
\text { of } \\
\text { TMS }\end{array}$ & $\begin{array}{l}\text { Affected } \\
\text { Hem }\end{array}$ & Age & Amax & Bsig & I1/2 & T1/2 & Tmax & Blin & I0 & $\mathrm{r}^{2}$ \\
\hline J.M & right & right & 72 & 0.1438 & 560505 & $-6 \mathrm{E}+05$ & $\mathrm{n} / \mathrm{a}$ & 22.79 & $\mathrm{n} / \mathrm{a}$ & $\mathrm{n} / \mathrm{a}$ & 0.000 \\
\hline J.M & left & right & 72 & 0.6802 & 2.6976 & 85.121 & 23.83 & 22.79 & 0.0613 & 79.568 & 0.917 \\
\hline D.K & left & right & 75 & 0.7747 & 0.219 & 85.096 & 28.37 & 28.02 & 0.7252 & 84.54 & 0.661 \\
\hline D.K & right & right & 75 & n/a & n/a & n/a & n/a & n/a & n/a & n/a & 0.000 \\
\hline L.B & left & right & 66 & 4.8982 & 2.7847 & 64.372 & 26.57 & 25.72 & 0.4127 & 58.435 & 0.979 \\
\hline L.B & right & right & 66 & 3.6596 & 2.8423 & 87.922 & 27.6 & 25.93 & 0.3053 & 81.936 & 0.994 \\
\hline O.D & left & right & 86 & 3.0255 & 23.113 & 75.965 & 25.7 & 25.85 & 0.0326 & 29.546 & 0.658 \\
\hline O.D & right & right & 86 & 0.8526 & 2.6632 & 48.901 & 26.55 & 26.31 & 0.0736 & 43.069 & 0.121 \\
\hline F.B & right & right & 75 & $n / a$ & $n / a$ & $n / a$ & $n / a$ & $n / a$ & $n / a$ & n/a & 0.000 \\
\hline F.B & left & right & 75 & 2.3998 & 9.2058 & 107.47 & n/a & 24.66 & 0.0509 & 85.64 & 0.976 \\
\hline I.C & right & right & 79 & 0.225 & -0.524 & 224.74 & n/a & n/a & n/a & n/a & 0.000 \\
\hline I.C & left & right & 79 & 0.7354 & 3.3262 & 91.324 & n/a & n/a & n/a & n/a & 0.000 \\
\hline M-T. M & right & right & 82 & 0.3281 & -18.36 & 106.7 & n/a & 29.05 & n/a & n/a & 0.045 \\
\hline M-T.M & left & right & 82 & 4.3523 & 8.3691 & 95.808 & 27.39 & 27.25 & 0.1287 & 78.927 & 0.945 \\
\hline M.LC & left & left & 86 & 0.7812 & 0.1003 & 69.3 & n/a & 24.48 & n/a & n/a & 0.000 \\
\hline M.LC & right & left & 86 & 3.4149 & 6.6094 & 110.42 & n/a & 25.97 & 0.0692 & 91.55 & 1.000 \\
\hline
\end{tabular}

\section{Affected Hemisphere}

Non Affected Hemisphere 
Due to the nature of some dose-response curves, not all parameters could be filled for each subject (Not available (n/a)). Ex: the average latency from MEPs within I $1 \frac{1}{2}+/-$ $5 \%\left(\mathrm{~T}_{1 / 2}\right)$ could not be calculated in subjects whose $\mathrm{I} 1 / 2$ is over $100 \%$.

\subsection{Amplitude}

\subsubsection{Amplitude Plots}

Dose- response curves followed a sigmoid function in control subjects (Figure 3). Derived parameters showed significant interindividual variability in absolute values but not in relative differences (figures $3,4,5$ ).

In patients, we compared parameters from contra-lesional hemispheres against affected hemispheres. This allowed us to view the differences in dose-response while controlling for naturally occurring interindividual differences.

In the Non-CST group, dose-response curves also followed a sigmoid function (Figure 4). In the CST group (Figure 5), unaffected hemispheres behaved like those in normal subjects and Non-CST patients. However, a sigmoid function could not be fitted to a majority (85\%) of hemispheres where the CST was affected by lesions in contrast to only 15\% in non-affected hemispheres. $\mathrm{P}=0.01$ (Fisher Exact test) (Table 4). 
Figure 3: Amplitude of MEPs in control group

K.R - Left hemispher mean amplitudes

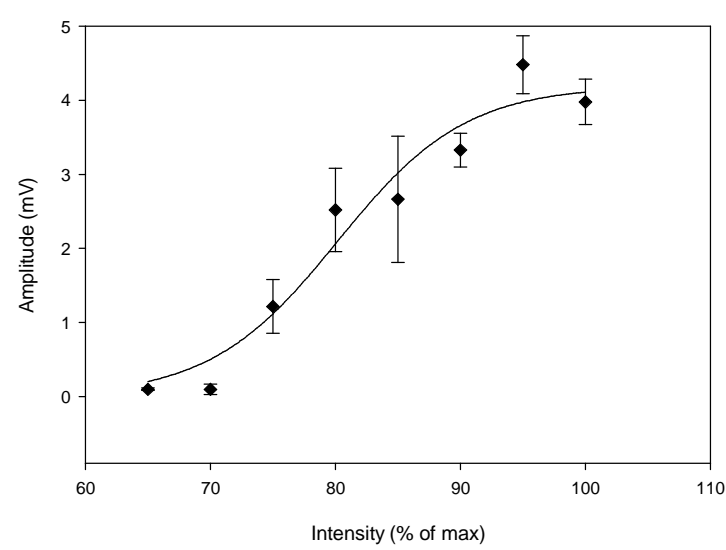

K.R - Right hemisphere mean amplitudes

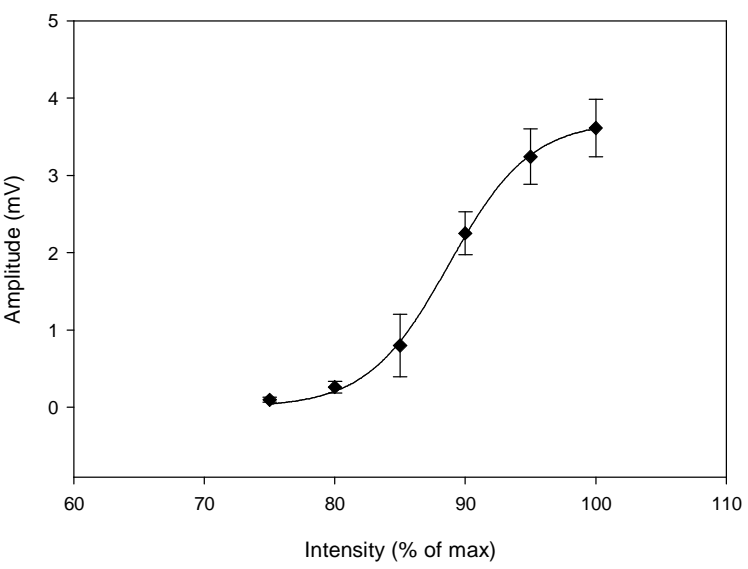

Figure 3: Example of TMS dose-response curves for the left and right hemispheres of a representative control subject. Amplitude, measured in millivolts $(\mathrm{mV})$ is represented on the $\mathrm{Y}$ axis. Intensity, measured as the percentage of maximum stimulator output, is represented on the $\mathrm{X}$ axis. Each point of the plot is the average amplitude of five stimulations at the same intensity. Error bars show the standard deviation of these points. These plots clearly demonstrate the sigmoid relationship found in dose-response curves of control subjects. 
Figure 4: Amplitude of MEPs in Non-CST group

PM - Non effected mean amplitudes



PM - Affected mean amplitudes

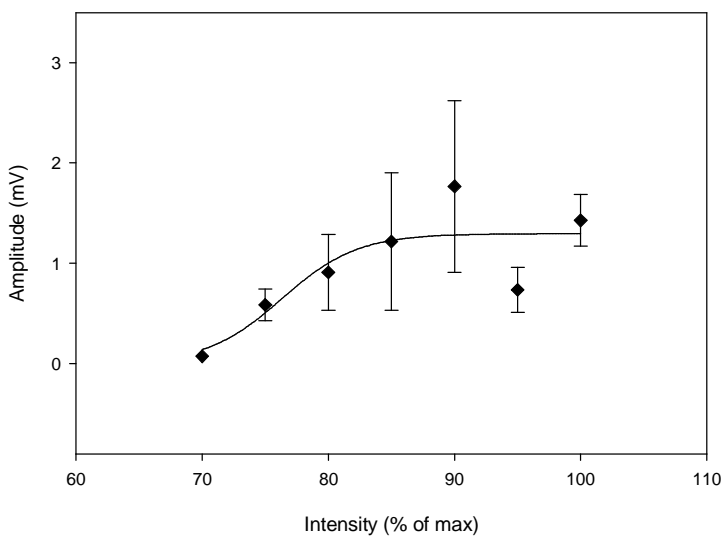

Figure 4: Example of TMS dose-response curves for the affected (right) and non-affected (left) hemispheres of a representative non-CST stroke patient. Features are as described in figure 3. These plots demonstrate the sigmoid relationship found in dose-response curves in the non-CST group despite having suffered subcortical strokes.

Figure 5: Amplitude of MEPs in CST group

J.M - affected mean amplitudes

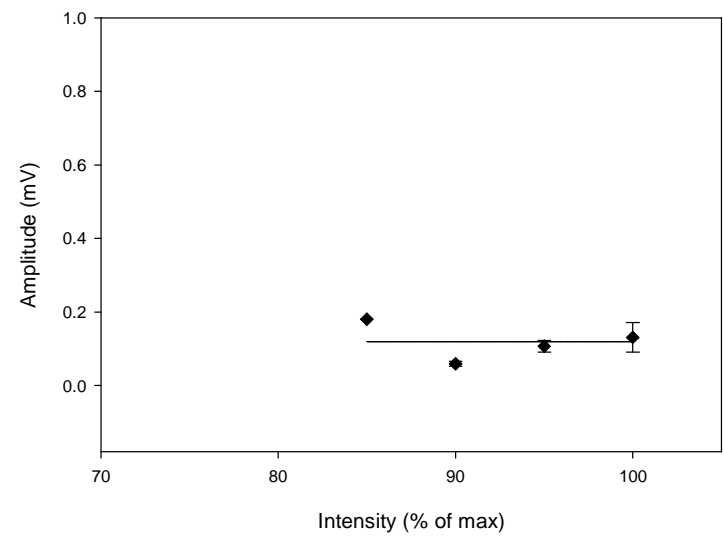

J.M - Non-affected mean amplitudes

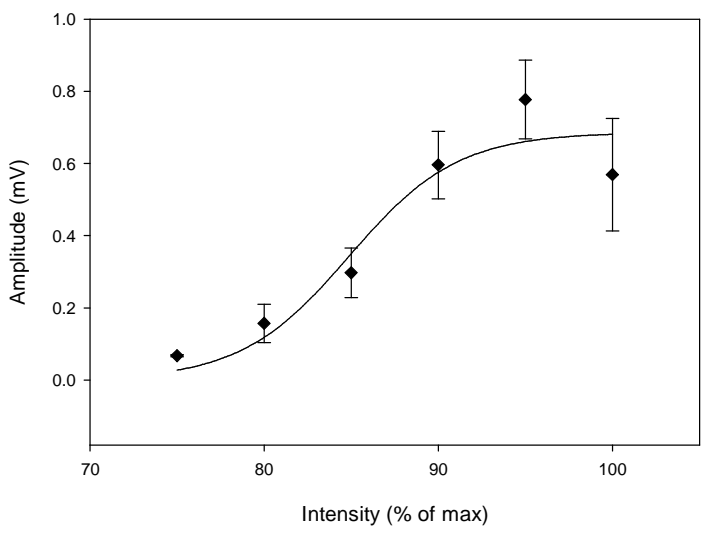

Figure 5: Example of TMS dose-response curves for the affected (left) and non-affected (right) hemispheres of a representative CST stroke patient. Features are as described in 
figure 3. These plots demonstrate the sigmoid relationship found in the dose-response curve of the non-affected hemisphere, and the absence of a sigmoid in the affected hemisphere of the CST group.

Table 4: Prevalence of Sigmoid Fit in CST Affected Stroke Patients

\begin{tabular}{|l|l|l|}
\hline & Fit & No Fit \\
\hline Affected & 1 & 7 \\
\hline Non - Affected & 7 & 1 \\
\hline
\end{tabular}

Table 4: A sigmoid function could not be fitted to a majority (87.5\%) of hemispheres affected by CST lesions in contrast to only $12.5 \%$ in non-affected hemispheres of patients in the CST group. $\mathrm{P}=0.01$ (Fisher Exact test).

\subsubsection{Statistical Analysis of Amplitude}

Statistical analysis revealed a trend in the amplitudes of the three groups when comparing the difference between the non-affected amplitude and the affected amplitude in patients (Figure 6), (ANOVA $\mathrm{P}=0.101)$. Since there was no statistical difference between the results obtained in the left and right hemispheres of control subjects, these were arbitrarily assigned as affected and non-affected, respectively for data analysis.

A pair-wise comparison indicated that this trend was caused by the CST group (Figure 7). When comparing between the hemispheres of the control group and the hemispheres of the Non-CST group, there were no statistical differences between the amplitudes of these hemispheres (Figure 7), (P > 0.05). However, in the CST group, the amplitudes of the affected hemispheres were lower than those of the non-affected hemispheres ( $\mathrm{P}=0.05$ corrected for multiple comparisons), (Figure 7). 
Figure 6: Difference in amplitude. Non-Affected - Affected

Difference in amplitude

Non-Affected - Affected

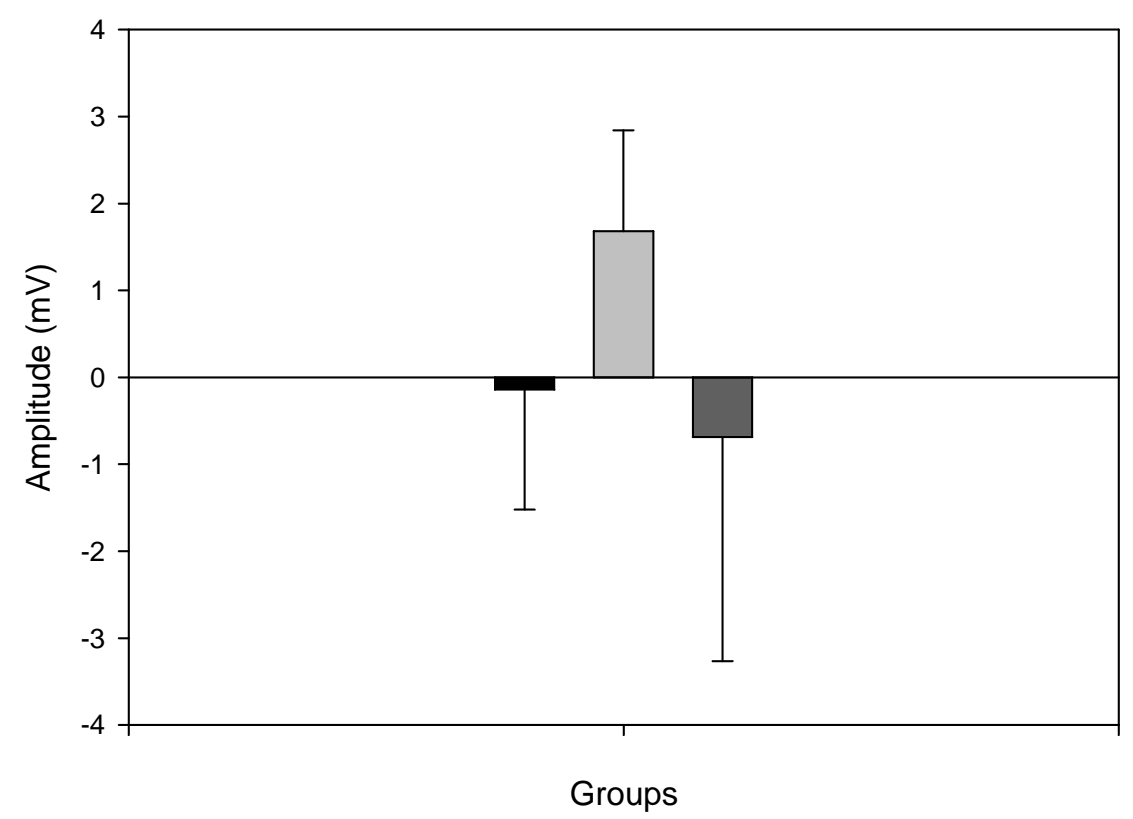

Control Group

Figure 6: Analysis of variance (ANOVA) of the difference in amplitude between the three observed groups. This analysis showed a statistical trend, $P=0.101$. The difference in amplitude, measured in millivolts $(\mathrm{mV})$ is represented on the $\mathrm{Y}$ axis. The control group (black), CST group (light grey) and Non-CST group (dark grey) are represented on the $\mathrm{X}$ axis. Error bars indicate standard deviation. 
Figure 7: Pair-wise comparison of amplitudes

a) Amplitudes of Control group

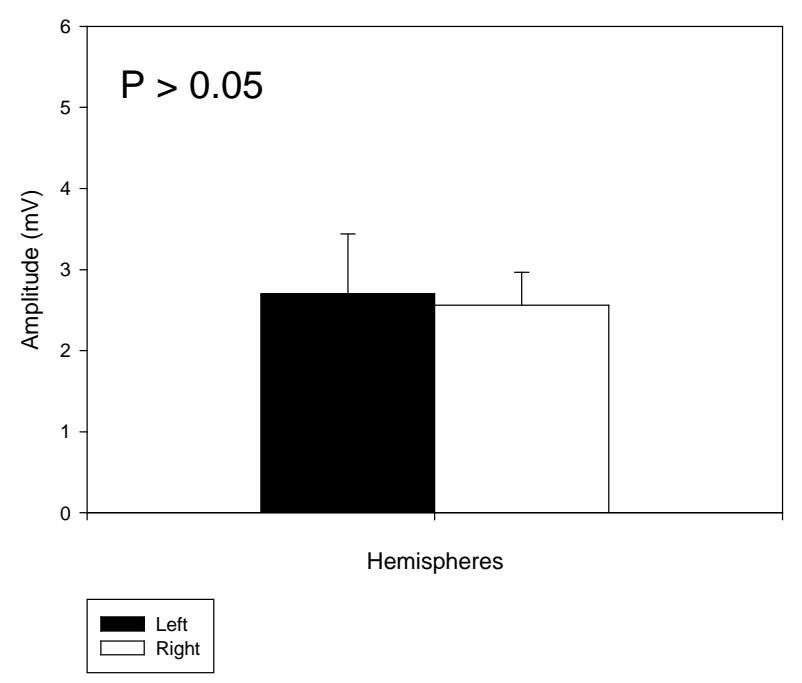

b) Amplitudes of Non-CST group

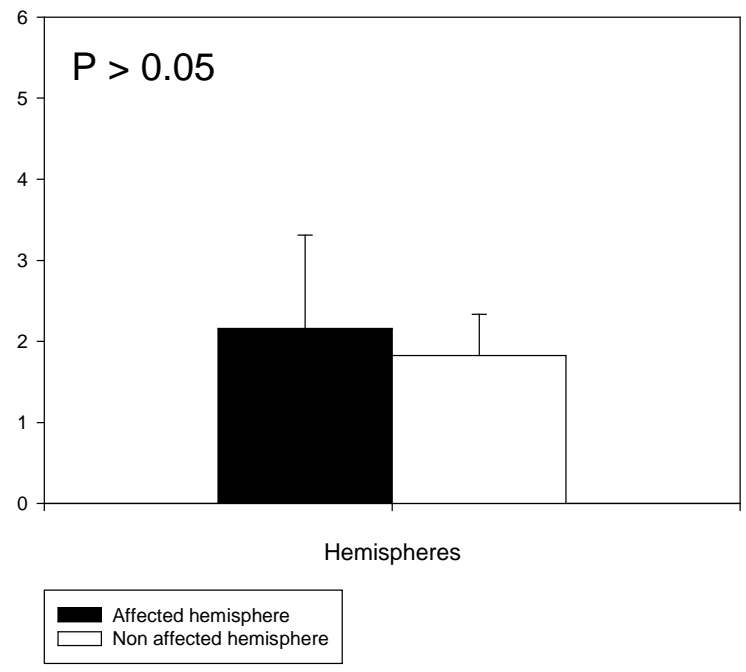


c)

Amplitudes of CST group

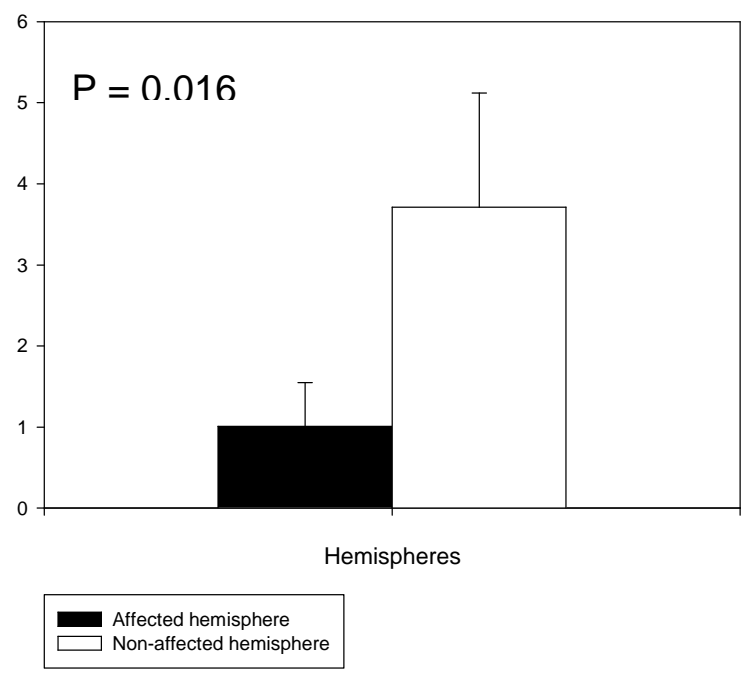

Figure 7: Pair-wise comparison between the hemispheres of: a) the control group, b) the Non-CST group and c) the CST group. Amplitude, measured in millivolts (mV) is represented on the $\mathrm{Y}$ axis. Hemispheres are represented on the $\mathrm{X}$ axis. Affected hemispheres of stroke patients and left hemispheres of controls are in black. Non-affected hemispheres of stroke patients and right hemispheres of controls are in white. Amplitudes of affected hemispheres in the CST group are significantly lower than the non-affected hemispheres $(\mathrm{P}=0.016)$. ( $\mathrm{P}=0.05$ when corrected for multiple comparisons $)$.

\subsection{Latency}

\subsubsection{Latency Plots}

Latency was plotted against stimulus intensity. Over all three groups, where a sigmoid shape is present in dose-response curves, latency becomes longer with decreasing intensity (Figures 8, 9, 10, 11, 12, 13). In the affected hemisphere of the CST group, where there is no steady recruitment phase of dose-response curves, similarly, latency showed no steady decrease (Figures 10, 13). 
Figure 8: Latency of MEPs in control group

P.D - Left hemisphere mean latencies

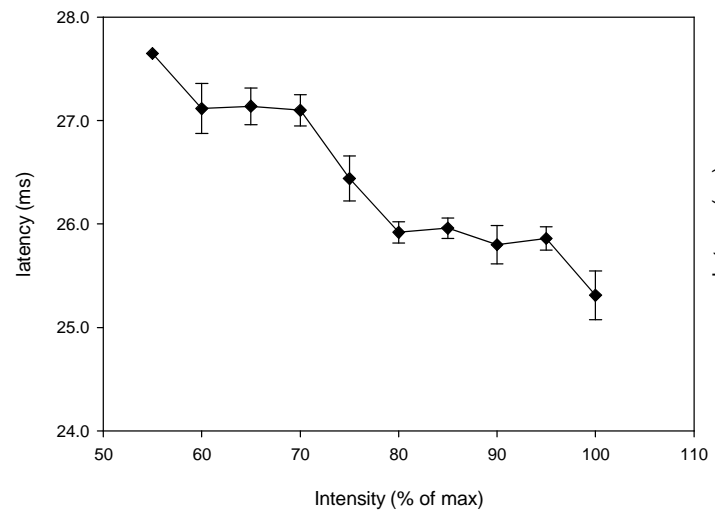

P.D - Right hemisphere mean latencies

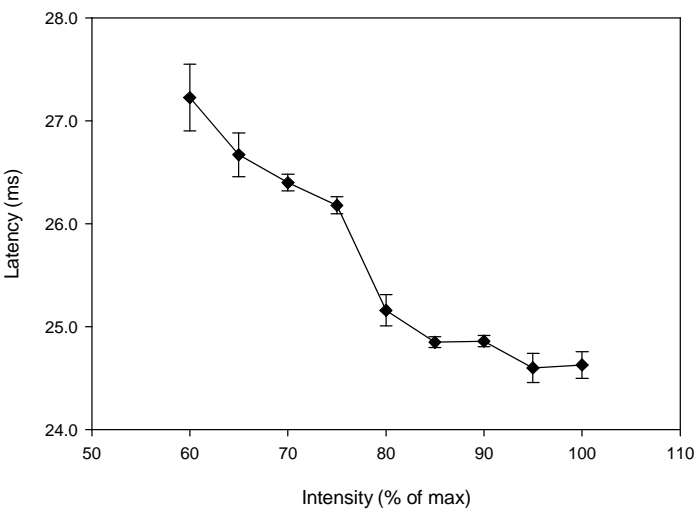

Figure 8: Example of latency curves for the left and right hemispheres of a representative control subject. Latency, measured in milliseconds (ms) is represented on the $\mathrm{Y}$ axis. Intensity, measured as the percentage of maximum stimulator output, is represented on the $\mathrm{X}$ axis. Each point of the plot is the average latency of five stimulations at the same intensity. Error bars show the standard deviation of these points.

Figure 9: Latency of MEPs in non-CST group

PM - Non affected average latencies

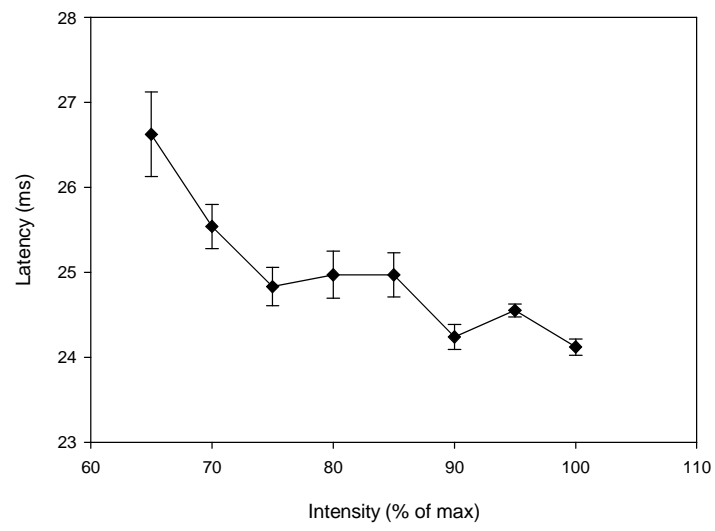

PM - Affected mean latency

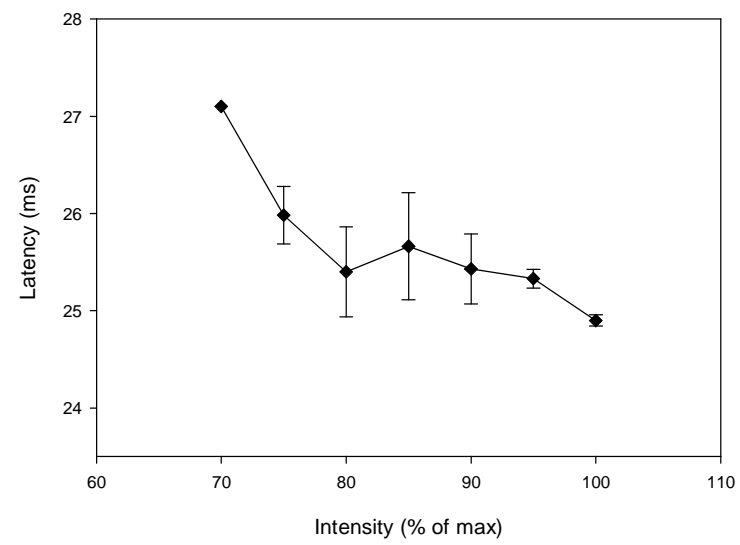


Figure 9: Example of latency curves for the affected (right) and non-affected (left) hemispheres of a representative non-CST stroke patient. Features are as described in figure 8 .

Figure 10: Latency of MEPs in CST group

J.M - Affected mean latencies



J.M - Non affected mean latencies

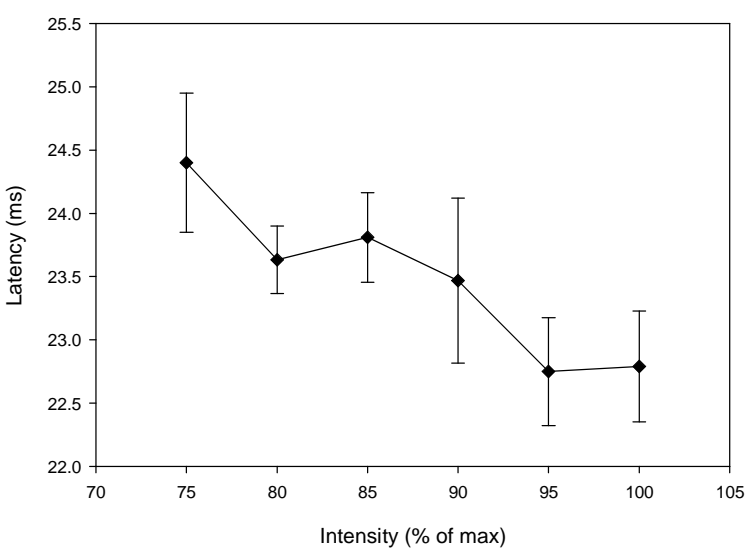

Figure 10: Example of latency curves for the affected (left) and non-affected (right) hemispheres of a representative CST stroke patient. Features are as described in figure 8.

Figure 11: Average latencies of Control Group

Average Latencies: Left Hemispheres of Control Group



Average Latencies: Right Hemispheres of Control Group




Figure 11: Average latencies of each stimulus intensity for the control group represented as a percentage of maximum latency. Maximum latency was defined as the average latency at $100 \%$ stimulus intensity. Latency, measured in percentage (\%) of the shortest latency at maximum stimulus intensity is represented on the $\mathrm{Y}$ axis. Intensity, measured as the percentage of maximum stimulator output, is represented on the $\mathrm{X}$ axis. Error bars show the standard error of these points.

Figure 12: Average latencies of Non-CST Group
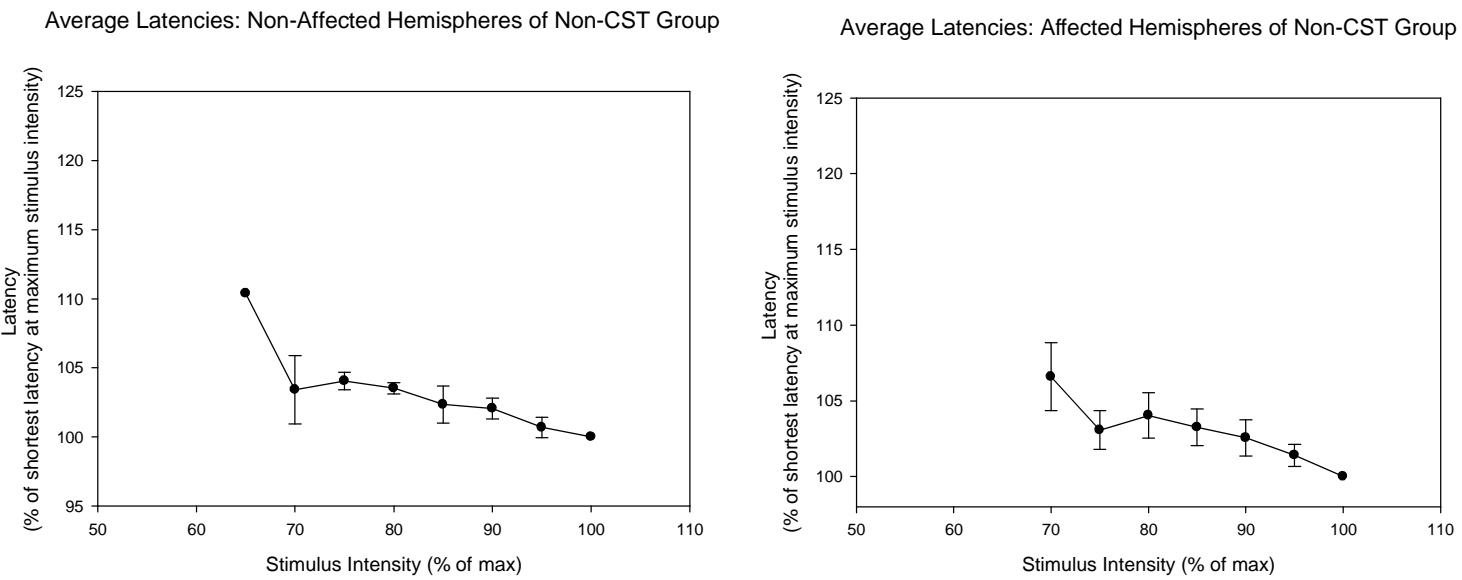

Figure 12: Average latencies of each stimulus intensity for the Non-CST group represented as a percentage of maximum latency. Features are as described in figure 11. 
Figure 13: Average latencies of CST Group

Average Latencies: Non-Affected Hemispheres of CST Group

Average Latencies: Affected Hemispheres of CST Group
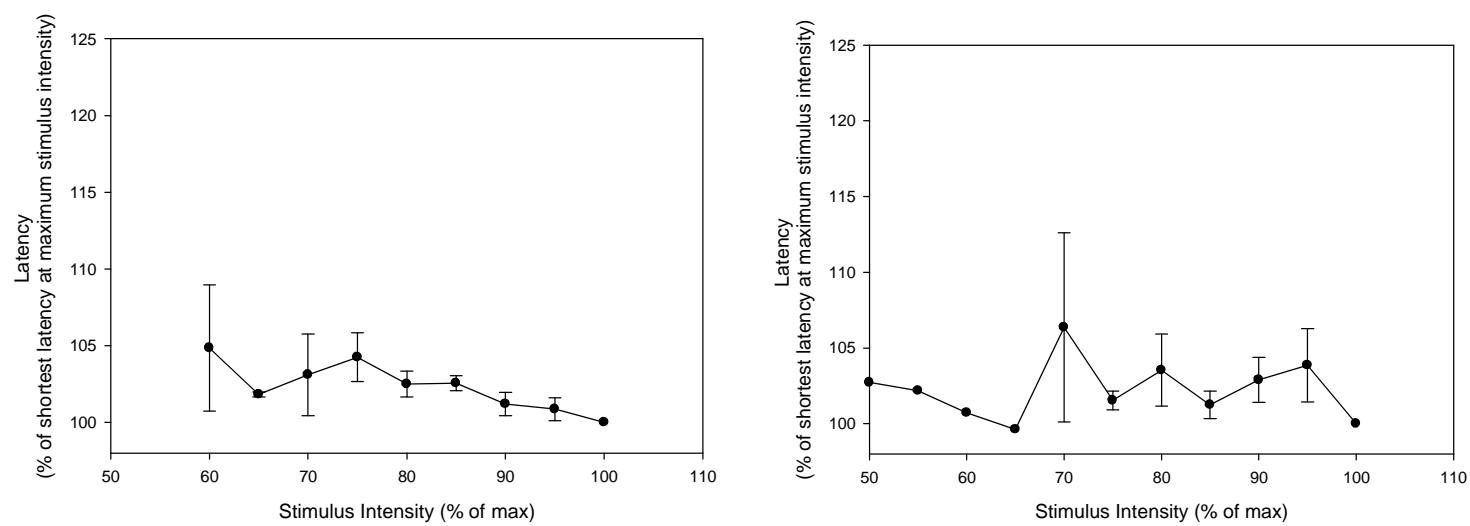

Figure 13: Average latencies of each stimulus intensity for the CST group represented as a percentage of maximum latency. Features are as described in figure 11.

\subsubsection{Statistical Analysis of Latency}

Statistical analysis revealed no significant differences between the latencies of any of the observed groups (ANOVA $\mathrm{P}=0.775$ ), (Figure 14). For this analysis, the average latency of the five TMS pulses delivered at 100\% stimulation intensity (average maximum latency) was compared between the affected hemisphere and the non affected hemisphere. The "Difference in Latency" is the value of the average maximum latency of the affected hemisphere subtracted from the average maximum latency of the nonaffected hemisphere. 
Figure 14: Difference in Latency. Non-Affected - Affected

Difference in Latency

Non-Affected - Affected

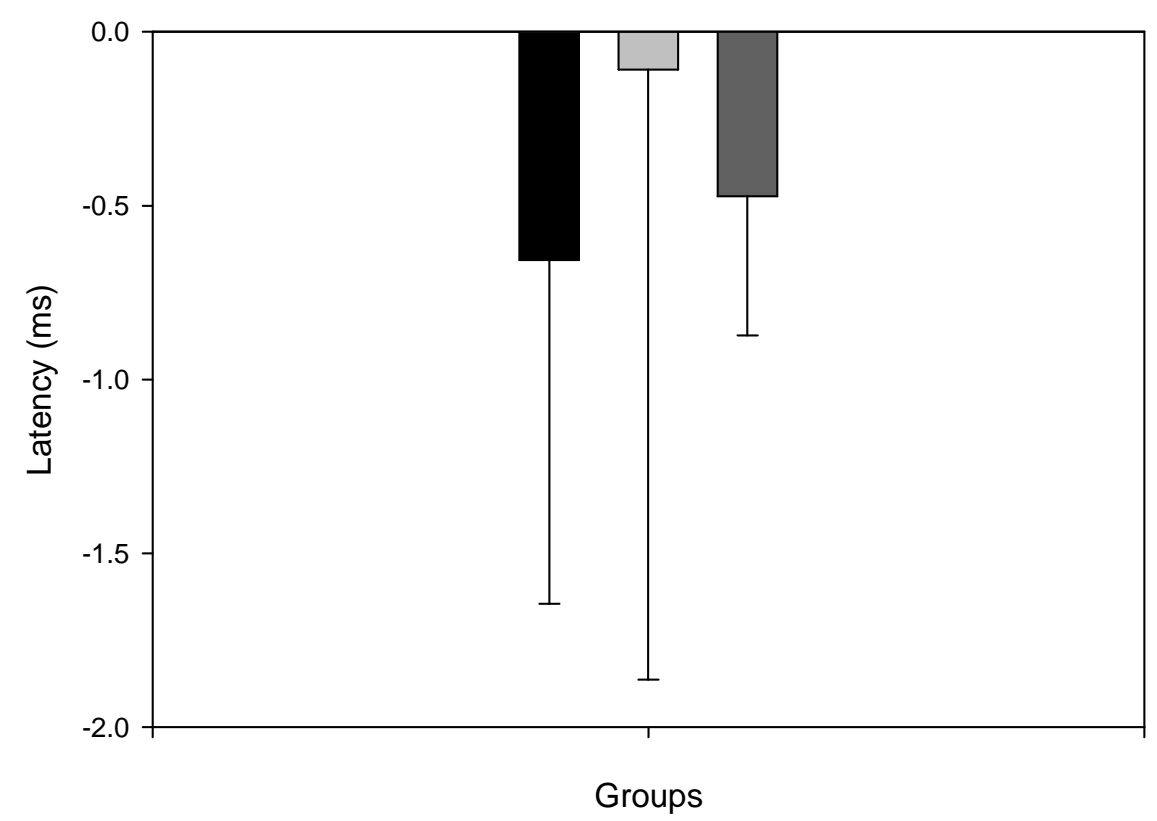

Control Group

CST Group

Non-CST Group

$$
\mathrm{P}=0.775
$$

Figure 14: Analysis of variance (ANOVA) of the difference in latency between the three observed groups. This analysis showed no statistical significance $\mathrm{P}=0.775$. The difference in latency, measured in milliseconds (ms) is represented on the $\mathrm{Y}$ axis. The control group (black), CST group (light gray) and Non-CST group (dark gray) are represented on the $\mathrm{X}$ axis. Error bars indicate standard deviation.

\subsection{Motor Threshold}

There was no statistical significance between the resting motor thresholds of any of the observed groups (ANOVA $\mathrm{P}=0.562$ ), (Figure 15). In addition, there were no statistical significance between the Extrapolated Motor Thresholds of the control group 
compared to the non-CST group (T-Test $\mathrm{P}=0.515$ ) (Figure16). In this last analysis, only the control group and the non-CST group were considered because we could not derive the EMT from the affected hemispheres of the CST group due to a lack of a sigmoid relationship. In these analyses, the interhemispheric differences of RMT and EMT were compared between each group.

Figure 15: Difference in Resting Motor Threshold. Non-Affected - Affected

Difference in RMT

Non-Affected - Affected

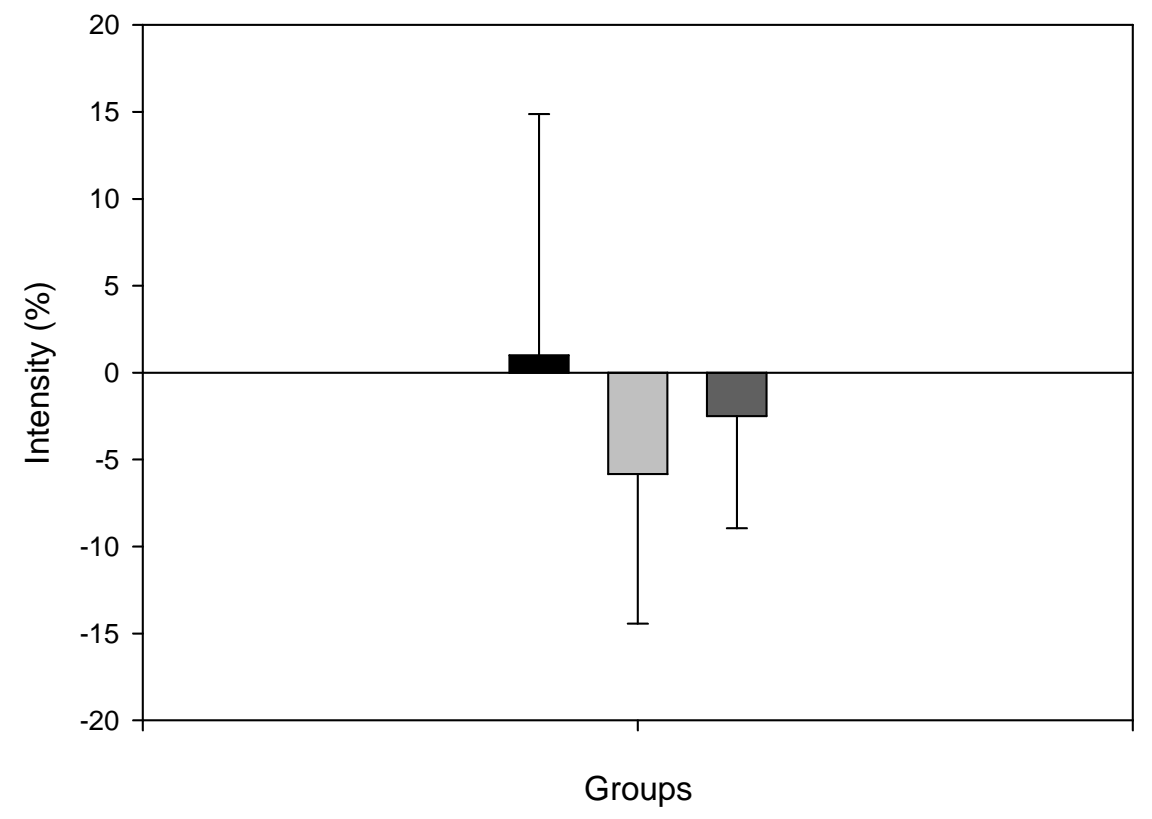

Control Group

$$
\mathrm{P}=0.562
$$

Figure 15: Analysis of variance (ANOVA) of the difference in resting motor threshold between the three observed groups. This analysis showed no statistical difference, $\mathrm{P}=$ 0.562. The difference in RMT, measured in percentage of maximum stimulator output (\%), is represented on the Y axis. The control group (black), CST group (light gray) and 
Non-CST group (dark gray) are represented on the $\mathrm{X}$ axis. Error bars indicate standard deviation.

Figure 16: Difference in Extrapolated Motor Threshold. Non-Affected - Affected

\author{
Difference in EMT \\ Non-Affected - Affected
}

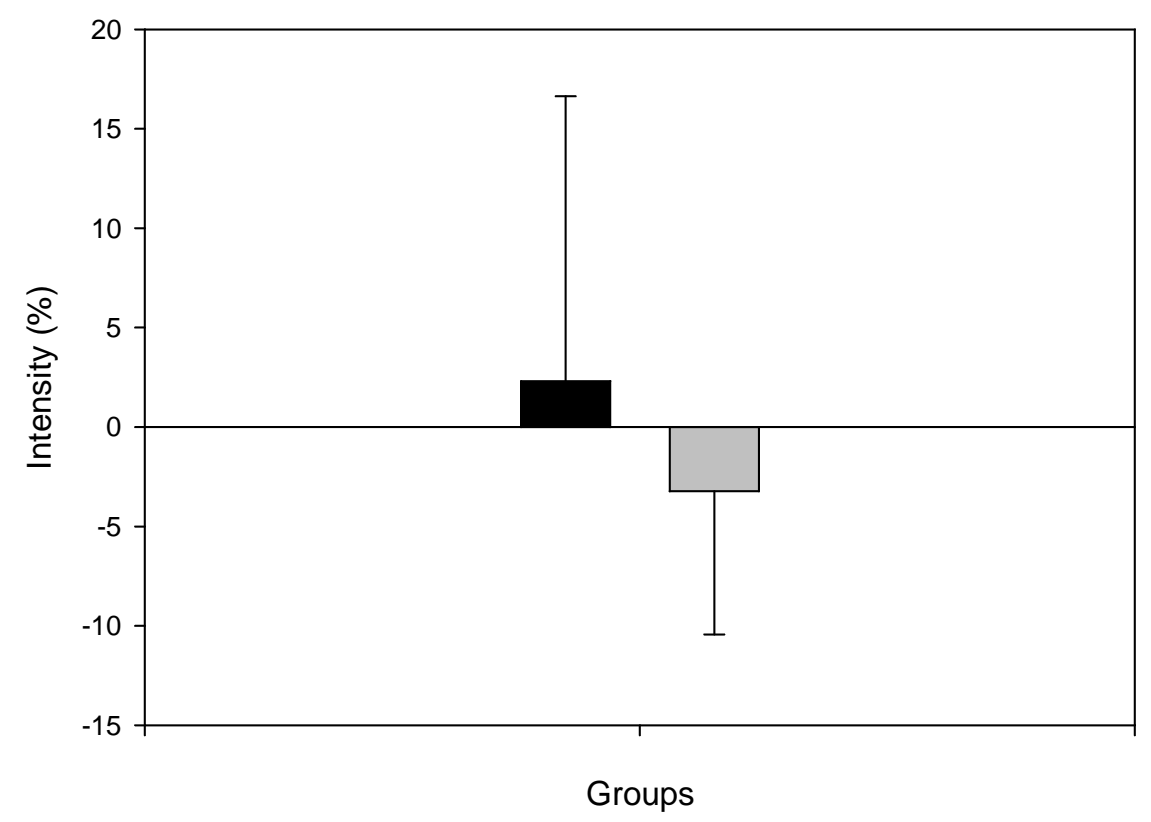

Control Group Non-CST Group

$$
\mathrm{P}=0.515
$$

Figure 16: Difference between the extrapolated motor thresholds of the control group and the non-CST group. T-Test shows no statistical difference, $\mathrm{P}=0.515$. The difference in EMT, measured in percentage of maximum stimulator output (\%), is represented on the $\mathrm{Y}$ axis. The control group (black) and Non-CST group (gray) are represented on the $\mathrm{X}$ axis. Error bars indicate standard deviation. 
In order address the question whether RMT and EMT give different or similar information about cortical excitability, RMT was plotted versus EMT (Figure 17). This plot indicated a non-linear relationship, which was best described using an exponential function $\left(y=e^{a x}\right)$ for unaffected hemispheres (Figure 17). There was no relationship, however, between the slope of the sigmoid function and the EMT

Figure 17: Relationship between Resting Motor Threshold and the Extrapolated Motor Threshold

\section{RMT vs EMT}

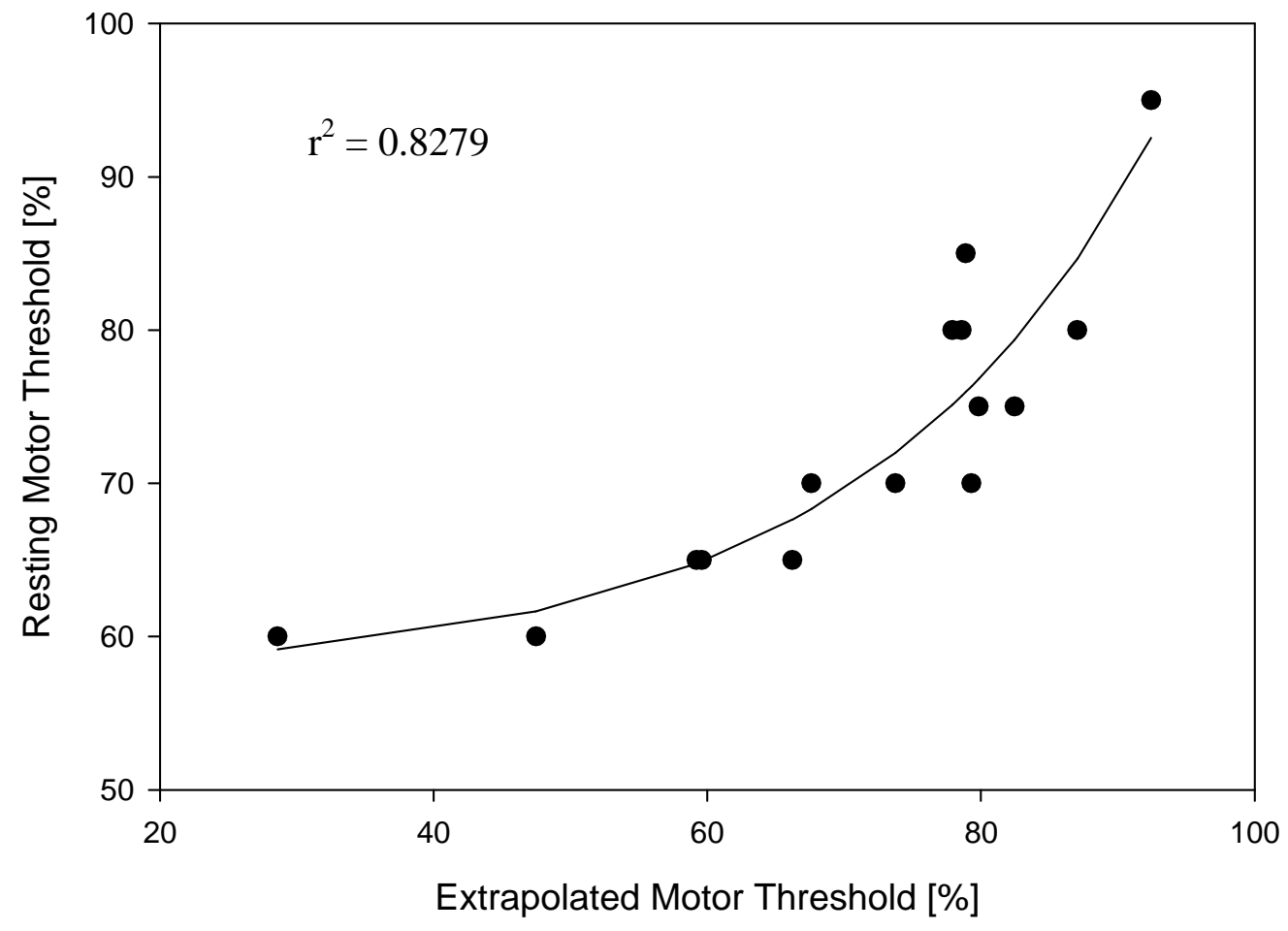

Figure 17: Exponential relationship between the resting motor threshold and the extrapolated motor threshold of non-affected hemispheres of all three groups. Both 
thresholds are measured as a percentage of maximum stimulator output. RMT is represented on the $\mathrm{Y}$ axis and EMT on the $\mathrm{X}$ axis. $\mathrm{r}^{2}=0.8279$

\subsection{Pooled Data Analysis}

Since the results of the above test did not show a significant difference between the Non-CST group and the control group with respect to the differences of amplitude, latency as well as the motor thresholds, these two groups were pooled together and compared to the CST group. T-tests showed a significant difference in the amplitudes (P $=0.033)$, but not in the latencies $(\mathrm{P}=0.486)$ or RMT $(\mathrm{P}=0.334)$ (Figure 18).

Figure 18: Pooled Non-CST and Control group compared to CST group
a)
Difference in Amplitude




Difference in Latenecy
Non-Affected - Affected
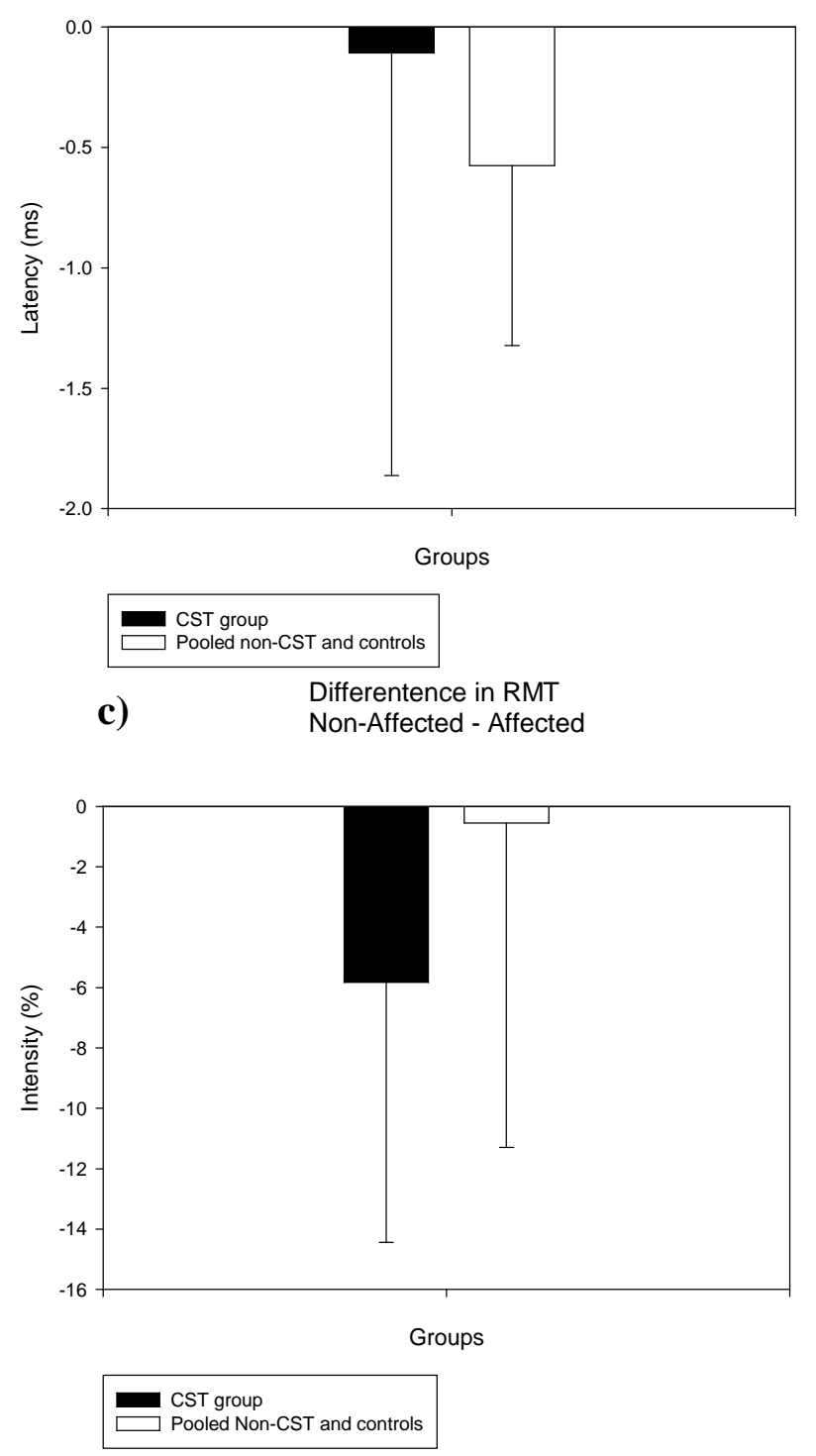

Figure 18: T-tests of pooled Non-CST and Control group data (white) compared to the CST group (black). Groups are represented on the $\mathrm{X}$ axis. a) The difference in amplitude, measured in millivolts $(\mathrm{mV})$ is represented on the $\mathrm{Y}$ axis $(\mathrm{P}=0.033)$. b) The difference in latency, measured in milliseconds $(\mathrm{ms})$ is represented on the $\mathrm{Y}$ axis $(\mathrm{P}=0.486)$. c) The difference in RMT, measured in percentage of maximum stimulator output $(\%)$, is represented on the $\mathrm{Y}$ axis $(\mathrm{P}=0.334)$. Error bars indicate standard deviation. 


\subsection{Relation of MEP parameters to motor function}

MEP amplitudes were compared to the behavioral motor scales in order to determine if there was a relationship. The percentage of change from one hemisphere to the other was used as a measure of relative amplitude to compare with scores on the Rivermead Motor Assessment and the Nine Hole Peg Test. There was no relationship between the relative amplitude and the motor assessments. There was also no strong correlation between the fit of the sigmoid and the motor assessments (rivermead $\mathrm{r}^{2}=$ 0.242; NHPT $\left.r^{2}=0.097\right)($ Table 5). This may be due to the fact that there was a ceiling effect in the motor assessments. Nearly all the observed patients performed well enough to fall within the same category. This effect can be due to the fact that patients who were too severely impaired to complete a certain level of these tests did not fit our inclusion criteria. In order to avoid a ceiling effect for patients well enough to participate in this study, more specific motor assessments should be used in future experiments.

Table 5: Summary of behavioral motor tests

$\begin{array}{cccccc}\text { Initials } & \text { Group } & \text { Age } & \begin{array}{c}\text { Rivermead arm } \\ (0 \text { (bad)- } \\ 15(\text { good) })\end{array} & \begin{array}{c}\text { 9-hole } \\ \text { Affected side } \\ \text { (time) }\end{array} & \begin{array}{c}r^{2} \\ \text { Fit of sigmoid } \\ \text { on affected } \\ \text { side }\end{array} \\ \text { LB } & \text { CST } & 66 & 14 & 32 \mathrm{sec} & 0.994 \\ \text { FB } & \text { CST } & 75 & 6 & \text { unable } & 0.000 \\ \text { IC } & \text { CST } & 79 & 14 & 22 \mathrm{sec} & 0.000 \\ \text { OD } & \text { CST } & 86 & 14 & 25 \mathrm{sec} & 0.121 \\ \text { DK } & \text { CST } & 75 & 12 & 40 \mathrm{sec} & 0.000 \\ \text { MLC } & \text { CST } & 86 & 8 & \text { unable } & 0.000 \\ \text { MTM } & \text { CST } & 82 & 14 & 26 \mathrm{sec} & 0.045 \\ \text { JM } & \text { CST } & 72 & 13 & 15 \mathrm{sec} & 0.000 \\ \text { DHA } & \text { NONCST } & 82 & 14 & 15 \mathrm{sec} & 0.698 \\ \text { SD } & \text { NONCST } & 49 & 15 & 15 \mathrm{sec} & 0.995 \\ \text { PM } & \text { NONCST } & 75 & 11 & \text { unable } & 0.696 \\ \text { RW } & \text { NONCST } & 78 & 15 & 20 \mathrm{sec} & 0.963 \\ \text { JB } & \text { NONCST } & 64 & 14 & 18 \mathrm{sec} & 0.995\end{array}$




\subsection{Influence of stimulus order on MEP dose-response curve parameters.}

As described in the methods section, the MEP dose-response curves were all recorded in the same way: At the area of maximum MEP response over the motorcortex, stimulation intensity was set to $100 \%$ and then decreased in $5 \%$ intervals. This allowed us to remain focused on the area of maximum response from the previous higher stimulation intensity. Starting with low stimulator output would have required a more extensive search in case the hotspot was lost at low stimulation intensities and would thus have prolonged the test sessions significantly. The protocol as described above already took 1.5 to $2 \mathrm{~h}$ per patient. Additional test would hardly have been tolerated by stroke patients.

In order to exclude that the stimulus order (starting with high intensity and going down to lower intensities) has an effect on the recorded dose-response curve, we did the following experiment in six young, healthy control subjects. These subjects were tested using the identical setup as described in 2.7. In half of them (3 subjects), dose responsecurves of the right motor cortex were recorded as described starting with $100 \%$ stimulation intensity and decreasing in 5\% intervals (top-down protocol). 5 pulsed were given at each intensity level and were separated by a time of 7-12 seconds. After completion of the top-down protocol, subjects had a short break in which they could move their heads and hands, but had to remain seated. Following the break, the doseresponse-curve was recorded again using the same hot-spot as before, this time starting with the minimum stimulator intensity, increasing in 5\% intervals to maximum intensity (bottom-up protocol). The other half of the subjects were investigated with the same setup but starting with the bottom-up protocol followed by the top-down protocol. For these three subjects, the hotspot was determined using $80 \%$ of the maximum stimulator output. 
The maximum amplitude of the sigmoid fit, EMT and the average latency of responses at the maximum intensity (max latency) were used as parameters for comparison. A paired T-test was performed for each of these three parameters to compare between the bottom-up and top-down protocol. There were no significant differences in any of the three parameters when comparing one protocol to the next $(\mathrm{P}>0.05)$.

Figure 19: Amplitude, EMT and latency compared in regular vs inverse protocol

a)

Amplitude

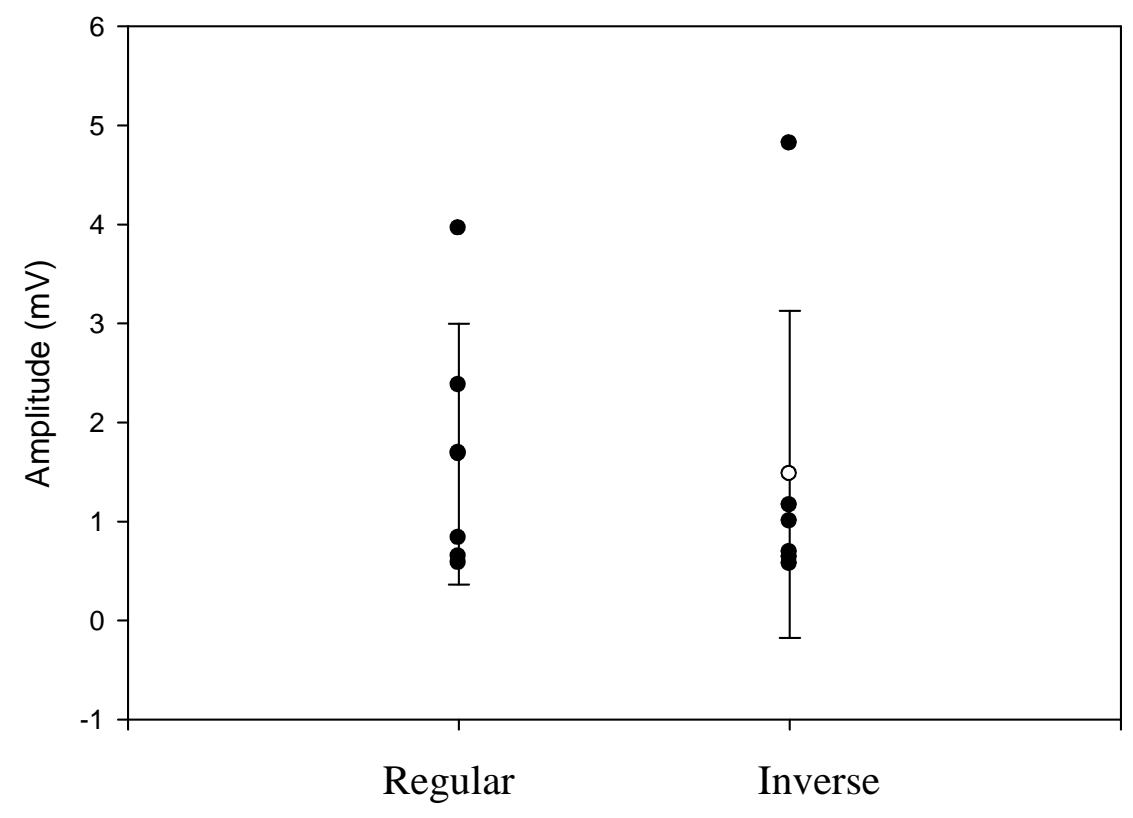


b)

\section{EMT}

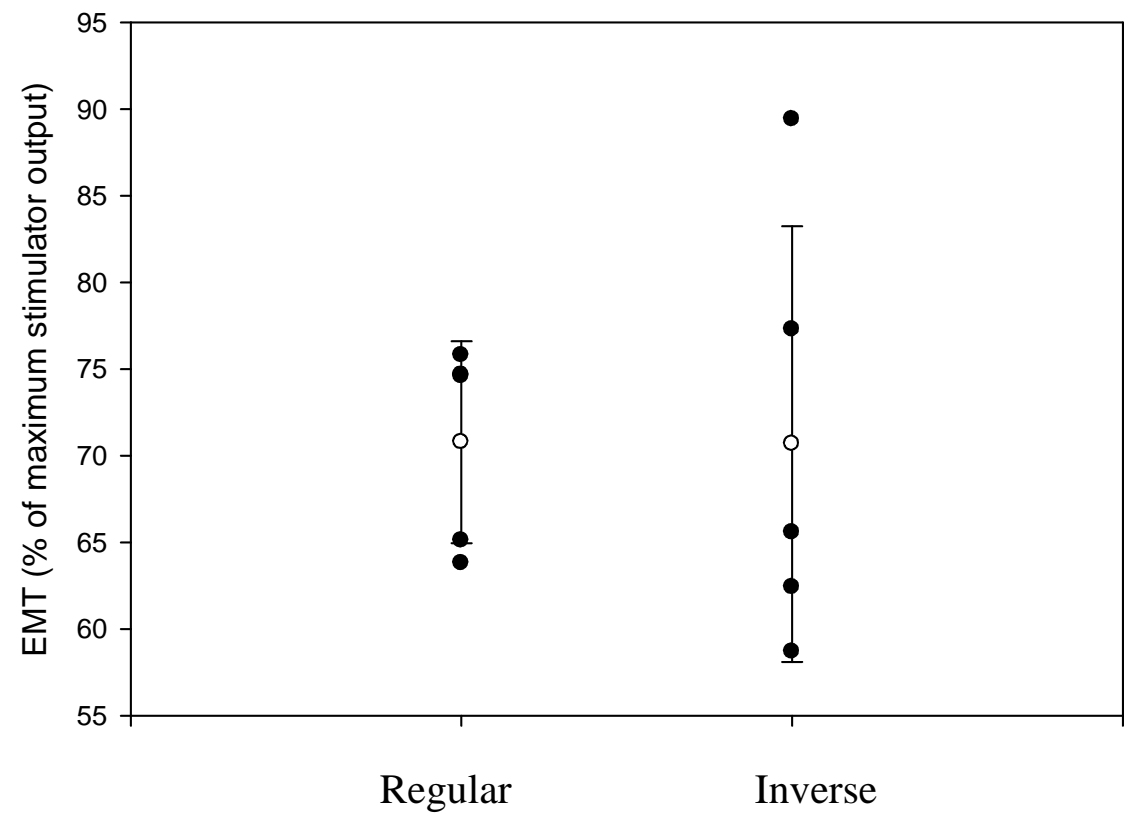

c)

\section{Latency}

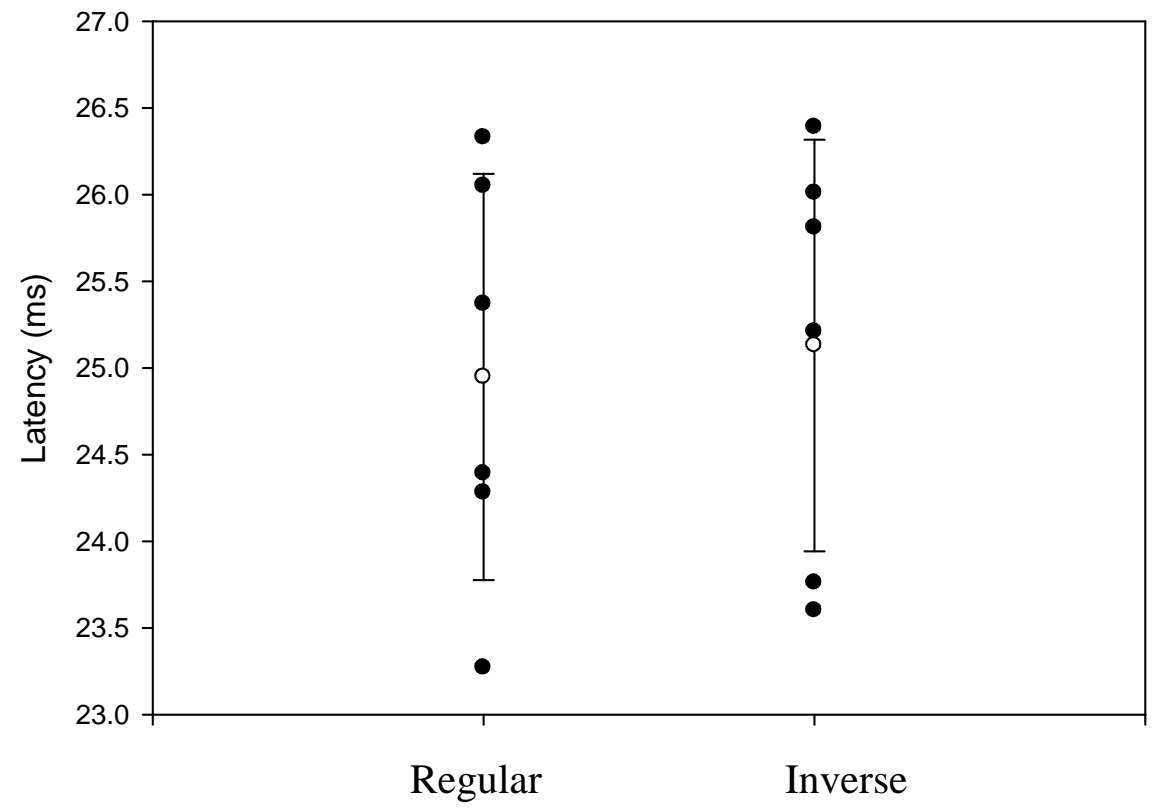


Figure 19: a) Maximum amplitude of 6 control subjects who received TMS as per the regular protocol described in the Methods section (Left) and with an inverse protocol (Right). Error bars show the standard deviation. Pair-wise comparison between the two methods showed no significant difference in amplitude $(\mathrm{P}>0.05)$. Amplitude, measured in $\mathrm{mV}$, is represented on the $\mathrm{Y}$ axis. Regular and inverse TMS protocols are represented on the $\mathrm{X}$ axis. b) EMT of the same subjects as in a). Pair-wise comparison between the two methods showed no significant difference in EMT $(\mathrm{P}>0.05)$. Furthermore, despite the difference in range of data points between the two methods, the goodness of fit of the sigmoid relationship $\left(\mathrm{r}^{2}\right)$ showed no significant difference in one method compared to the other $(\mathrm{P}>0.05)$. EMT, measured in percentage $(\%)$ of maximum stimulator output, is represented on the $\mathrm{Y}$ axis. All other parameters are as described for a). c) Maximum latency of the same subjects as in a) and b). Pair-wise comparison between the two methods showed no significant difference in latency $(\mathrm{P}>0.05)$. Maximum latency, measured in milliseconds (ms), is represented on the $\mathrm{Y}$ axis. All other parameters are as described for a).

\subsection{Summary of Results}

- A sigmoid function could not be fitted to a majority (85\%) of hemispheres affected by CST lesions in contrast to only $15 \%$ in non-affected hemispheres. $\mathrm{P}=$ 0.01 (Fisher Exact test).

- Comparison of amplitude between 3 groups indicated a trend towards a difference $\mathrm{P}=0.10($ ANOVA) 
- This trend is caused by the CST group which had higher differences in amplitudes than the control groups. Pair-wise comparisons $(\mathrm{P}=0.05$ corrected for multiple comparisons).

- No statistical significance between the latencies of any of the observed groups. $\mathrm{P}=$ 0.775 (ANOVA).

- No statistical significance was found between the resting motor thresholds of any of the observed groups. $\mathrm{P}=0.562$ (ANOVA).

- No statistical significance between the Extrapolated Motor Thresholds of the control group vs non-CST group. $\mathrm{P}=0.515$ (T-Test).

- No relationship between relative amplitude and motor function tests.

- Significant exponential relationship between EMT and RMT for unaffected hemispheres.

- There are no significant differences in parameters of dose-response curves when the order of TMS pulses is reversed. 


\section{Chapter 4}

\section{DISCUSSION \& CONCLUSION}




\section{Discussion}

\subsection{Amplitude}

The amplitude of dose-response curves correlates with the number of motor-units that are recruited in the muscle by the stimulation. It serves as an indirect measure of the number of fibres stimulated (Brouwer \& Schryburt-Brown, 2006). Lesions in the CST had a direct effect on the amplitude of dose-response curves. It can thus be concluded that that the number of axons, available for conducting actions potentials is reduced and that fewer neurons are recruited by TMS (Van der Kamp et al., 1996; Krings, Naujokat \& Keyserlingk, 1998; Luft et al., 2001; Paine et al., 2006; Werhahn et al., 2007).

Lesions in other subcortical areas, that were not directly affecting the CST did not affect amplitude and hence not affect the number of neurons recruited by TMS. The recruitment of neurons in the pyramidal tract of non-CST patients was the same as in healthy controls. There was no evidence that non-CST subcortical lesions affect motor neuron excitability through modulation of other descending pathways. We can confirm that subcortical lesions that are not affecting the CST have no affect on cortical excitability or fiber tract integrity. These results are consistent with previous reports (Liepert, Hamzel, \& Weiller, 2000; Shimizu et al., 2002; (Butefisch et al., 2003).

In CST-affected patients the maximum amplitude was lower when compared to non-CST affected patients and controls. Thus, with the same stimulus intensity, a lower number of neurons were recruited in CST patients. This is caused by localized ischemic damage of neurons in the CST (Stinear et al., 2007). This phenomenon altered the recruitment process of pyramidal tract neurons in CST patients.

In all control subjects and 7 out of 8 patients where the CST was not affected, recruitment of CST neurons through TMS was represented by a sigmoid curve. The 
recruitment pattern of a sigmoid curve was characterized by a slow beginning, followed by a steep increase (linear portion) and finished by leveling off (ceiling effect). The underlying mechanisms of a sigmoid recruitment are thought to be caused by the interaction of excitatory and inhibitory neurons (Di Lazzaro et al. 2004).

Inhibitory interneurons and excitatory motorneurons are both stimulated by TMS and MEPs reflect the net sum of these inputs onto pyramidal cells. However, inhibitory interneurons have a lower threshold for recruitment and saturate earlier than excitatory motorneurons (Peterson, Pyndt \& Nielsen, 2003; Werhahn et al., 2007). Therefore the slow initial non-linear part of motorneuron recruitment seen in sigmoid curves were due to the relatively high recruitment of inhibitory interneurons and low recruitment of excitatory motor neurons at low stimulus intensities.

Once the maximum number of interneurons has been recruited, the inhibitory effect on the motorneurons can no longer be increased. At the same intensity however, due to their higher thresholds, additional motorneurons can still be recruited with increasing stimulation intensity. This behaviour is results in the steep increase in recruitment of excitatory motorneurons characterized by the linear portion of the sigmoid curve (Werhahn et al., 2007). Since this linear part is clearly dominated by recruitment of motor-neurons, it was supposed to be a better source for parameters measuring cortical excitability. Thus the EMT (in contrast to the RMT) was thought to better describe the intensity where the inhibitory effect of interneurons ended and the unrestricted recruitment of excitatory neurons began.

The ceiling effect of the sigmoid occurred when the intensity of the stimulation was high enough to recruit all available neurons in the target area. For example, when all fibers pertaining to the FDI muscle were recruited at an intensity of $85 \%$ of the maximum 
stimulator output, higher intensities would not produce larger amplitudes. They were only able to match the amplitudes attained at the $85 \%$ intensity level.

The sigmoid relationship was reliably fitted to dose-response curves of TMS in normal controls (Figure 3). These results are consistent with previous studies (Luft et al., 2001; Tranulis et al., 2006; Malcolm et al. 2006; Van Kuijk et al., 2008). Subcortical stroke patients whose lesions were not directly affecting the CST (Non-CST group) had the same recruitment pattern as normal controls (Figure 4) indicating that subcortical lesions per se have little impact on cortical excitability. The sigmoid shape was absent in cases where lesions were directly affecting the CST (CST-group) (Figure 5). In these patients, neurons in the pyramidal tract followed an all-or nothing recruitment pattern.

In controls, as the stimulus intensity increased, so did the number of recruited neurons. In CST patients however, a lesion on the tract impeded the recruitment of subsequent neurons despite the rising intensity. As the intensity of the stimulation increased, there was not necessarily an increase in recruited neurons. The inconsistency of the recruitment of neurons in CST patients depends on the size and exact location of the lesion on the tract (Stinear et al., 2007; Yamamoto, Raisman \& Li, 2009).

Although CST patients did not exhibit a sigmoid relationship in their doseresponse curves, the amplitude of their threshold recruitment (max amplitude) was reliably measured. The maximum amplitude offered a means of comparison from one hemisphere to the other regardless of the absence or presence of a sigmoid recruitment pattern. 


\subsection{Latency}

Latency is typically used to derive measures of conduction velocity (PascualLeone et al., 2002; Peterson, Pyndt \& Nielsen, 2003). Conduction velocity is expected to be influenced in diseases and disorders affecting the myelin sheath around axons (Pascual-Leone et al., 2002). In stroke, lesions on the CST completely severe some axons, however they do not affect the myelin sheath of surviving axons (Li et al., 2009). Therefore, the time which it takes these surviving axons to relate information from the cell body to their peripheral synapses is not affected (Lasiene et al., 2008). There may be a decrease in functioning axons, which is reflected in a drop of amplitude, however the intact axons will conduct action potentials with normal velocities (Lasiene et al., 2008).

The relationship between latency and stimulus intensity was not affected by subcortical stroke, regardless of the location of the lesion. As the stimulus intensities decreased, the MEP latencies increased. These results are consistent with previous findings (Van der Kamp et al., 1996; Krings, Naujokat \& Keyserlingk, 1998; Luft et al., 2001; Paine et al., 2006). With increasing stimulus intensities, a larger number of motor neurons are recruited (Krings, Naujokat \& Keyserlingk, 1998; Luft et al., 2001; Paine et al., 2006). Newly recruited neurons may be better myelinated and hence be more efficient and have shorter latencies than previously activated neurons (Van der Kamp et al., 1996; Paine et al., 2006). In addition, stimulation at higher intensities results in a more direct response from excitatory motorneurons since there is a saturation of inhibitory interneuron activity (Van der Kamp et al., 1996; Peterson, Pyndt \& Nielsen, 2003). 


\subsection{Methods of determining motor threshold}

The relationship between the RMT and the EMT was non-linear. We observed a positive exponential relationship between the two methods of determining MT (Figure 17). This correlation indicated an increase in sensitivity of the EMT at lower stimulation intensities. This implies that EMT is able to discriminate cortical excitability in subjects at the lower end of the intensity scale, where RMT would suggest the same level of cortical excitability over a relatively wide range of stimulation intensities and thus tend to underestimated MT. It suggests that EMT may be more suitable for patients with a low MT. EMT should therefore be used for subjects with high cortical excitability.

In our patient population, this method was limited by the necessity to record a sigmoid function in dose-response curves in order to determine MT. As demonstrated with our data this was not the case in patients where the CST is affected.

EMT might be better suited for patients who suffer from neurodegenerative diseases affecting motor systems. Although these patients would be expected to have altered cortical excitability, they do not suffer an acute structural impairment of the pyramidal tracts (Martorana et al., 2008). If a reliable fit of a sigmoid function can be attained in the dose-response curves, the EMT may reveal information about motorneuron excitability at low stimulation intensities where no MEPs fulfilling the requirements for RMT can be derived. This might be the case in Parkinson's disease or in amyotrophic lateral sclerosis.

\subsection{Effect of Stimulation Order}

The effect of stimulation order was tested by comparing parameters obtained through our top-down methods with parameters from a bottom-up protocol. The 
maximum amplitude and latency as well as EMT were collected from six control subjects. The variability of the parameters between one protocol and the other were lower than the normally occurring variability observed in TMS (Butefisch et al., 2003). Although they were not significant, there was some disparity in these parameters from one protocol to the next and the limited power of the tests calls for a cautious interpretation of the absence of a significant effect. However, in order to determine the effect of the order of TMS pulse intensities, approximately 100 subjects would be necessary to obtain a sufficiently powered test. For example, the difference of the amplitudes between one protocol and the next had a mean of 0.2 and standard deviation of 0.7 . Therefore, in order for a change of 0.2 to be detected with an expected standard deviation of change at 0.7 , the desired power of the test being 0.8 and the alpha error at 0.05, the sample size would have to be 99 subjects. This on the other hand illustrates that the clinical importance of such differences (even if they were significant in a sufficiently powered experiment) are probably low.

\subsection{Hypothesis Tested}

The first part of the tested hypothesis stated that dose-response curves of patients with subcortical stroke would also follow a sigmoid function. Results indicated that this is not entirely the case. Dose-response curves did not follow a sigmoid function in CST affected stroke patients. They did, however, follow a sigmoid function in subcortical stroke not affecting the CST and in control subjects.

The second part of the hypothesis stated that parameters derived from doseresponse curves were related to the severity of clinical impairment. This statement was also only partially true. We were not able to demonstrate a significant correlation 
between parameters of dose-response curves and measures of motor-function impairment. One reason for this might be the limited sensitivity of motor function tests in patients with mild to moderate impairment because we observed a ceiling effect in both behavioral tests.

Despite this, pyramidal tract impairment can be determined in most patients ( 7 out of 8) by the absence or presence of a sigmoid relationship of dose-response curves. In those patients where a sigmoid can not be produced, we can presume that there is marked CST impairment.

\subsection{Future Applications}

In clinical use, amplitudes of an affected hemisphere are measured at 10\%-20\% above the motor threshold of the unaffected hemisphere and these are used as a descriptor of cortical excitability (Schippling et al.2009; Brouwer \& Schryburt-Brown, 2006). Our study suggests the error will be large if the patient's recruitment process on their affected side follows an upper threshold characteristic. $10 \%-20 \%$ of stimulator output above the RMT of a non-affected hemisphere may not yet attain the threshold recruitment intensity of the affected hemisphere (Figure 20 a). Tests done in this way may produce results that do not accurately reflect the level of impairment of the patient.

Another method used clinically to assess cortical excitability is to set a fixed amplitude, and determine what level of stimulation is required to attain that amplitude (Sale \& Ridding, 2007). Finding the intensity of a fixed unaffected amplitude is difficult because is may be sub-threshold on the affected side (Figure $20 \mathrm{~b}$ ). Using any amplitude lower than the maximum as a means of comparison between a healthy and an affected 
hemisphere may be difficult due to the upper-threshold recruitment process of affected hemispheres.

In order to compare one hemisphere to the next, it is suggested that amplitudes should be measured at the upper inflection point of the sigmoid function in unaffected hemispheres (Figure $20 \mathrm{c}$ ). Consequently, the maximum amplitudes should primarily be used as a parameter for comparison between hemispheres.

Figure 20: Recommendations for the use of TMS dose response curves

a)

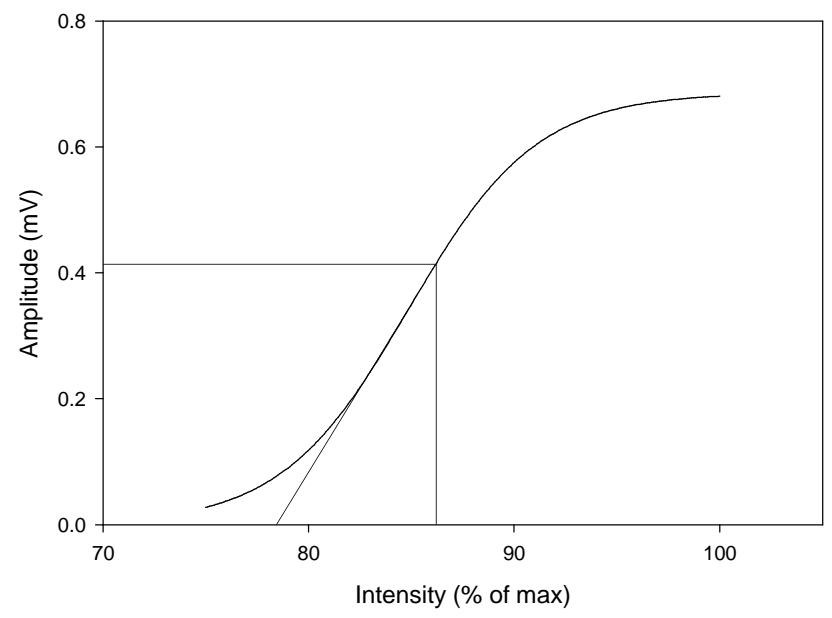

b)

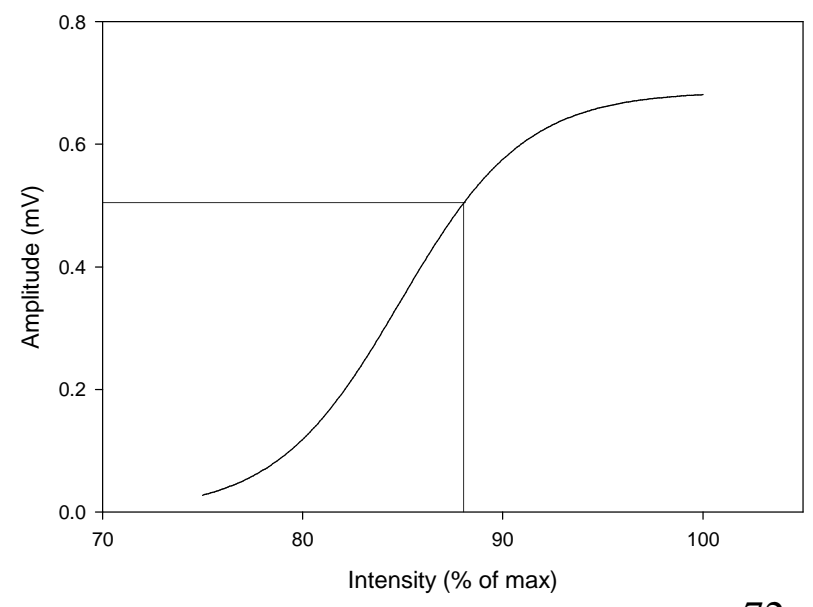




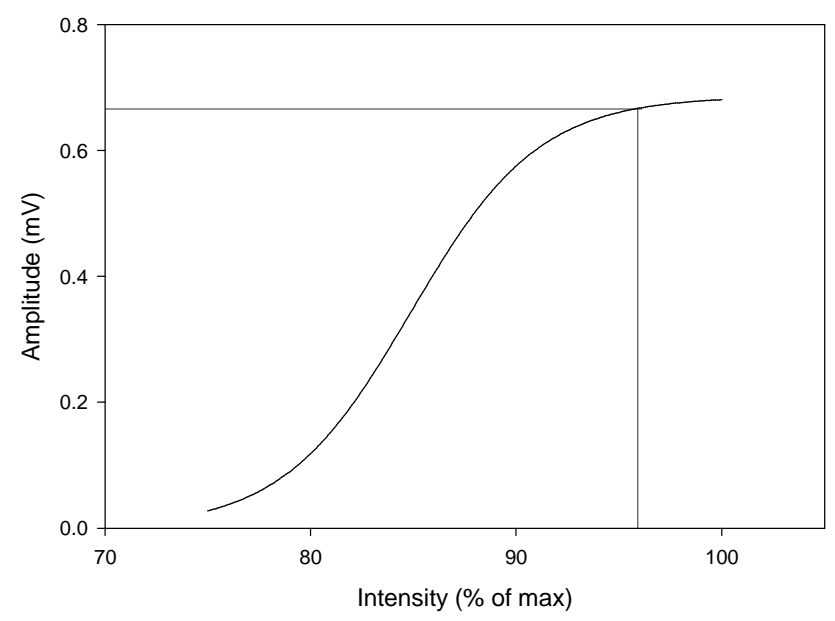

Figure 20: Example of TMS dose-response curves of a healthy hemisphere. Amplitude, measured in millivolts $(\mathrm{mV})$ is represented on the $\mathrm{Y}$ axis. Intensity, measured as the percentage of maximum stimulator output, is represented on the $\mathrm{X}$ axis. a) EMT is shown as the tangent to the steepest section of the sigmoid curve. Lines are drawn to indicate the intensity and amplitude at $110 \%$ of the motor threshold. In cases where a CST affected hemisphere demonstrates an upper-threshold recruitment, $110 \%$ of this motor threshold will not be sufficient to as a means of comparison. Intensity at $110 \%$ of motor threshold is not sufficient to reach the upper threshold of CST affected hemispheres (Upper infection point of the sigmoid curve). b) Lines are drawn to indicate the intensity needed in order to obtain a predetermined amplitude $(0.5 \mathrm{mV})$. Any predetermined amplitude of a non-affected hemisphere short of the maximum amplitude may be insufficient as a means of comparison with CST affected hemispheres that follow an upper-threshold recruitment. c) Lines are drawn to show the amplitude and intensity of the upper inflection point of the sigmoid function. This point, determined in healthy hemispheres is a valuable point of comparison with CST affected hemispheres. It is a value that can be 
compared irrespective of the type of neuron recruitment during the stimulation of a hemisphere.

EMT and other derived parameters, such as the slope of the sigmoid, may be useful characteristics in the assessment of cortical excitability and CST integrity in neurodegenerative diseases. These diseases progress over a period of time and can offer a means of understanding the relationship between derived parameters of dose-response curves and clinical impairment. These may prove to be a valuable method of studying these relationships rather than incidents that have an acute onset and produce an all-ornone response to TMS. In brief, TMS is a promising technique for the future investigation of motor injury and recovery, among other neuroscientific topics.

\subsection{Conclusion}

In conclusion, a sigmoid function best described dose response curves in healthy controls of all ages. This coincides with the findings of previous studies. The sigmoid relationship was also present in patients who have suffered a subcortical stroke that was not directly affecting the CST. In CST-affected stroke patients, a sigmoid relationship was no longer present. These patients exhibited an all-or-nothing recruitment of excitable neurons, rather than a sigmoid-type recruitment seen in other groups.

The maximum amplitude of dose-response curves can be used to compare affected hemispheres to non-affected hemispheres of a subject. Absolute amplitudes should not, however be used as a means of comparison between individuals due to a high level of interindividual variability. 
Other parameters of MEPs or dose-response curves, such as latency, or the slope of the sigmoid function offered no further information on the integrity of the CST. The relationship between the traditional RMT and the novel EMT indicated the latter might have advantages as to sensitivity when evaluating subjects that have low motor thresholds. These two methods showed little difference in subjects with high motor thresholds. 


\section{REFERENCE LIST}

1. Adams, S.A., Ashburn, A., Pickering, R.M. \& Taylor, D. (1997). The scalability of the rivermead motor assessment in acute stroke patients. Clinical Rehabilitation, $11.42-51$.

2. Ahonen, J. P., Jehkonen, M., Dastidar, P., Molnar, G., \& Hakkinen, V. (1998). Cortical silent period evoked by transcranial magnetic stimulation in ischemic stroke. Electroencephalogr.Clin.Neurophysiol., 109, 224-229.

3. Alagona, G., Delvaux, V., Gerard, P., De, P., V, Pennisi, G., Delwaide, P. J. et al. (2001). Ipsilateral motor responses to focal transcranial magnetic stimulation in healthy subjects and acute-stroke patients. Stroke, 32, 1304-1309.

4. Anderson, K.D., Gunawan, A. \& Steward, O. (2005). Quantitative assessment of forelimb motor function after cervical spinal cord injury in rats: Relationship to the cortocospinal tract. Experimental Neurology, 194. $161-174$.

5. Bernad, D. M. \& Doyon, J. (2008). The role of noninvasive techniques in stroke therapy. Int.J.Biomed.Imaging, 2008, 672582.

6. Berweck, S., Walther, M., Brodbeck, V., Wagner, N., Koerte, I., Henschel, V. et al. (2008). Abnormal motor cortex excitability in congenital stroke. Pediatr.Res., $63,84-88$.

7. Brouwer, B. J. \& Schryburt-Brown, K. (2006). Hand function and motor cortical output poststroke: are they related? Arch.Phys.Med.Rehabil., 87, 627-634. 
8. Bueche, F.J. (1988). Principles of Physics $-5^{\text {th }}$ Edition. McGraw-Hill Book Co. 406- 461

9. Butefisch et al. (2003). Remote changes in cortical excitability after stroke. Brain, 126. $470-481$.

10. Butefisch, C. M., Wessling, M., Netz, J., Seitz, R. J., \& Homberg, V. (2008). Relationship between interhemispheric inhibition and motor cortex excitability in subacute stroke patients. Neurorehabil.Neural Repair, 22, 4-21.

11. Cacioppo, J., Tassinary, L.G. \& Berntson, G.G. (2007). The Handbook of Psychophysiology, $3^{\text {rd }}$ Edition. New York, NY: Cambridge University Press. 124125.

12. Caramia, M. D., Palmieri, M. G., Giacomini, P., Iani, C., Dally, L., \& Silvestrini, M. (2000). Ipsilateral activation of the unaffected motor cortex in patients with hemiparetic stroke. Clin.Neurophysiol., 111, 1990-1996.

13. Chen, H-M. et al. (2009). Test-retest reproducibility and smallest real difference of 5 hand function tests in patients with stroke. Neurorehabilitation and Neural Repair, $23435-440$.

14. Chen, R., Tam, A., Butefisch, C., Corwell, B., Ziemann, U., Rothwell, J. C. et al. (1998). Intracortical inhibition and facilitation in different representations of the human motor cortex. J.Neurophysiol., 80, 2870-2881.

15. Cizek, J. et al. (2004). Fast and robust registration of PET and MR images of human brain. Neuroimage, 22. $434-442$. 
16. Cowey, A. (2005). The Ferrier Lecture 2004 what can transcranial magnetic stimulation tell us about how the brain works? Philos.Trans.R.Soc.Lond B Biol.Sci., 360, 1185-1205.

17. Danner, N., Julkunen, P., Kononen, M., Saisanen, L., Jouko, N. \& Karhu, J. (2008) Navigated transcranial magnetic stimulation and computed electric field strength reduce stimulator-dependent differences in the motor threshold. J. of Neuroscience Methods 174, 116-122.

18. De Gennaro, L. et al. (2007). Neurophysiological correlates of sleepiness:A combined TMS and EEG study. NeuroImage 33, 1277-1287.

19. Di Lazzaro, V. et al. (2004). The physiological basis of transcranial motor cortex stimulation in conscious humans. Clinical Neurophysiology, 115. $255-266$.

20. Di Lazzaro, V., Pilato, F., Dileone, M., Profice, P., Capone, F., Ranieri, F. et al. (2008). Modulating cortical excitability in acute stroke: a repetitive TMS study. Clin.Neurophysiol., 119, 715-723.

21. Duque, J., Mazzocchio, R., Dambrosia, J., Murase, N., Olivier, E., \& Cohen, L. G. (2005). Kinematically specific interhemispheric inhibition operating in the process of generation of a voluntary movement. Cereb.Cortex, 15, 588-593.

22. Escudero, J. V., Sancho, J., Bautista, D., Escudero, M., \& Lopez-Trigo, J. (1998). Prognostic value of motor evoked potential obtained by transcranial magnetic brain stimulation in motor function recovery in patients with acute ischemic stroke. Stroke, 29, 1854-1859. 
23. Foltys, H., Krings, T., Meister, I. G., Sparing, R., Boroojerdi, B., Thron, A. et al. (2003). Motor representation in patients rapidly recovering after stroke: a functional magnetic resonance imaging and transcranial magnetic stimulation study. Clin.Neurophysiol., 114, 2404-2415.

24. Greenstein, B. \& Greenstein, A. (2000). Colot Atlas of Neuroscience. Thieme. New York, NY. 180-185

25. Hendelman, W.J. (2006). Atlas of functional Neuroanatomy. Taylor \& Francis Group, LLC. Boca Raton, FL. 122-126

26. Herwig, U. et al. (2001). The navigation of transcranial magnetic stimulation. Psychiatry Research: Neuroimaging Section, 108. 123 - 131.

27. Hughes, C.J. \& John, N.W. (2003). A flexible infrastructure for delivering augmented reality enabled transcranial magnetic stimulation. University of Wales, Bangor. $1-6$.

28. Jahanshahi, M. \& Rothwell, J. (2000). Transcranial magnetic stimulation of cognition: an emerging field. Exp. Brain Res. 131: 1-9.

29. Johansen-Berg, H., Rushworth, M. F., Bogdanovic, M. D., Kischka, U., Wimalaratna, S., \& Matthews, P. M. (2002). The role of ipsilateral premotor cortex in hand movement after stroke. Proc.Natl.Acad.Sci.U.S.A, 99, 1451814523.

30. Kanagal, S.G. \& Muir, G.D. (2007). Bilateral dorsal funicular lesions alter sensorimotor behaviour in rats. Experimental Neurology, 205. 513 - 524.

31. Kim, Y. H., Jang, S. H., Byun, W. M., Han, B. S., Lee, K. H., \& Ahn, S. H. (2004). Ipsilateral motor pathway confirmed by combined brain mapping of a 
patient with hemiparetic stroke: a case report. Arch.Phys.Med.Rehabil., 85, 13511353.

32. Krings, T., Naujokat, C. \& Keyserlingk, D.G. (1998). Representation of cortical motor function as revealed by stereotaxic transcranial magnetic stimulation. Electroencephalography and clinical Neurophysiology, 109. 85 - 93.

33. Lasiene, J., Shupe, L., Perlmutter, S. \& Horner, P. (2008). No evidence for chronic demyelination in spared axons after spinal cord injury in a mouse. The Journal of Neuroscience, 28(15). 3887 - 3896.

34. Li, L. et al. (2009). MRI indentification of white matter reorganization enhanced by erythropoietin treatment in a rat model of focal ischemia. Stroke, 40.936941.

35. Liepert, J., Hamzel, F. \& Weiller, C. (2000). Motor cortex disinhibition of the unaffected hemisphere after acute stroke. Muscle Nerve 23, $1761-1763$.

36. Liepert, J., Storch, P., Fritsch, A., \& Weiller, C. (2000). Motor cortex disinhibition in acute stroke. Clin.Neurophysiol., 111, 671-676.

37. Luft, A.R. et al. (2001). Transcranial magnetic stimulation in the rat. Exp Brain Res, 140. $112-121$.

38. Makni, S., Beckmann, C., Smith, S. \& Woolrich, M. (2008). Bayesian deconvolution fMRI data bilinear dynamical systems. Neuroimage, 42. 1381 1396.

39. Malcolm, M.P., Triggs, W.J., Light, K.E., Shechtman, O., Khandekar, G., Gonzalez Rothi, L.J. (2006). Reliability of motor cortex transcranial magnetic 
stimulation in four muscle representations. Clinical Neurophysiology 117, 10371046

40. Martorana, A. et al. (2008). L-dopa modulated motor cortex excitability in Alzheimer's disease. J Neural Transm. 115. 1313 - 1319.

41. McIntire, M., Richter, W., Morden, D., Wennerberg, A. \& Frankenstein, U. (2003). Blood oxygen level dependent functional magnetic resonance imaging. Concepts in Magnetic Resonance 16A(1), 5 - 15.

42. McMillan, A.S., Watson, C., Walshaw, D. (1998). Improved reproducibility of magnetic stimulation-evoked motor potentials in the human masseter by a new method for locating stimulation sited on the scalp. Archives of Oral Biology 43, 665-668.

43. Mehta, C.R. \& Patel, N.R. (1983). A network algorithm for performing Fisher's Exact Test in r x c contingency tables. Journal of the American Statistical Association. Vol 78, No 382. $427-434$.

44. Muellbacher, W., Facchini, S., Boroojerdi, B., \& Hallett, M. (2000). Changes in motor cortex excitability during ipsilateral hand muscle activation in humans. Clin.Neurophysiol., 111, 344-349.

45. Netter, F.H., Craig, J.A., Perkins, J., Hansen, J.T. \& Koeppen, B.M. (2002). Atlas of Neuroanatomy and Neurophysiology. Icon Custom Communications, Teterboro, NJ. 70-72. 
46. Pascual-Leone, A., Davey. N. J., Rothwell, J., Wassermann, E. M. \& Puri, B. K. (2002). Handbook of Transcranial Magnetic Stimulation. New York, NY: Oxford University Press Inc.

47. Paine, P.A., et al. (2006). Assessing the temporal reproducibility of human esophageal motor-evoked potentials to transcranial magnetic stimulation. Journal of Clinical Neurophysiology, 23. 4. $374-380$.

48. Peterson, N.T., Pyndt, H.S. \& Nielsen, J.B. (2003). Investigating human motor control by transcranial magnetic stimulation. Exp. Brain Res 152, 1-Reis, J., Swayne, O. B., Vandermeeren, Y., Camus, M., Dimyan, M. A., Harris-Love, M. et al. (2008). Contribution of transcranial magnetic stimulation to the understanding of cortical mechanisms involved in motor control. J.Physiol, 586, $325-351$.

49. Renner, C. I., Woldag, H., Atanasova, R., \& Hummelsheim, H. (2005). Change of facilitation during voluntary bilateral hand activation after stroke. J.Neurol.Sci., $239,25-30$.

50. Sale, M.V. \& Ridding, M.C. (2007). Factors inXuencing the magnitude and reproducibility of corticomotor excitability changes induced by paired associative stimulation. Exp. Brain Res 181, 615 -626.

51. Schippling, S. et al. (2009). Abnormal Motor Cortex Excitability in Preclinical and Very Early Huntington's Disease. Biol Psychiatry 65, 959-965.

52. Schonfeldt-Lecuona, C. et al. (2005). Accuracy of stereotaxic positioning of transcranial magnetic stimulation. Brain Topography, 17. 4. 253 - 259. 
53. Shimizu, T. et al. (2002). Motor cortical disinhibition in the unaffected hemisphere after unilateral cortical stroke. Brain, 125. 1896 - 1907.

54. Smith, S., Jenkinson, M., Beckmann, C., Miller, K. \& Woolrich, M. (2007). Meaningful design and contrast estimability in fMRI. Neuroimage, 34. 127 - 136.

55. Sohn, Y. H. \& Hallett, M. (2004). Motor evoked potentials. Phys.Med.Rehabil.Clin.N.Am., 15, 117-31, vii.

56. Sommerfeld, D.K. et al. (2004). Spasticity after stroke: Its occurrence and association with motor impairments and activity limitations. Stroke, 35. $134-$ 139.

57. Sparing, R., Buelte, D., Meister, I.G., Paus, T. \& Fink, G.R. (2008). Transcranial magnetic stimulation and the challenge of coil placement: a comparison of conventional and stereotaxic neuronavigational strategies. Human Brain Mapping, 29. $82-96$.

58. Stinear, C. M., Barber, P. A., Smale, P. R., Coxon, J. P., Fleming, M. K., \& Byblow, W. D. (2007). Functional potential in chronic stroke patients depends on corticospinal tract integrity. Brain, 130, 170-180.

59. Tranulis, C., Gueguen, B., Pham-Scottez, A., Vacheron, M.N., Cabelguen, G., Costantini, A., Valero, G. \& Galinovski., A. (2006). Motor threshold in transcranial magnetic stimulation: comparison of three estimation methods. Clinical Neurophysiology 36, 1-7.

60. Traversa, R., Cicinelli, P., Pasqualetti, P., Filippi, M. \& Rossini, P.M. (1998). Follow-up of interhemispheric differences of motor evoked potentials from the 
'affected' and 'unaffected' hemispheres in human stroke. Brain Research 803, 1-

8.

61. Traversa, R., Cicinelli, P., Filippi, M., Oliveri, M., Palmieri, M. G., Pasqualetti, P. et al. (1999). A method to monitor motor cortical excitability in human stroke through motor evoked potentials. Brain Res.Brain Res.Protoc., 4, 44-48.

62. Van der Kamp et al. (1996). Cortical excitability and response variability of transcranial magnetic stimulation. American Clinical Neurophysiology Societey. Vol 13(2). $164-171$.

63. Van Kuijk, A. A., Pasman, J. W., Geurts, A. C., \& Hendricks, H. T. (2005). How salient is the silent period? The role of the silent period in the prognosis of upper extremity motor recovery after severe stroke. J.Clin.Neurophysiol., 22, 10-24.

64. Van Kujik, A.A. et al. (2008). Stimulus-response characteristics of motor evoked potentials and silent periods in proximal and distal upper-extremity muscles. Journal of Electromyography and Kinesiology, 19.4. $574-583$.

65. Werhahn, K.J., Behrang-Nia, M., Bott, M.C. \& Klimpe, S. (2007). Does the recruitment of excitation and inhibition in the motor cortex differ? Journal of Clinical Neurophysiology. Vol 24, No 5.419-423.

66. Werhahn, K. J., Conforto, A. B., Kadom, N., Hallett, M., \& Cohen, L. G. (2003). Contribution of the ipsilateral motor cortex to recovery after chronic stroke. Ann.Neurol., 54, 464-472. 
67. Woldag, H., Lukhaup, S., Renner, C., \& Hummelsheim, H. (2004). Enhanced motor cortex excitability during ipsilateral voluntary hand activation in healthy subjects and stroke patients. Stroke, 35, 2556-2559.

68. Yamamoto, M., Raisman, G. \& Li, Y. (2009). Loss of directed fore-limb reaching after destruction of spinal grey matter. Brain Research, 1265. 47 - 52

69. Zhang, W., Olivi, A., Hertig, S.J., van Zijl, P. \& Mori, S. (2008). Automated fiber tracking of human brain white matter using diffusion tensor imaging. NeuroImage $42,771-777$. 